\title{
Recovery of 3-D Structure From Motion Is Neither Euclidean Nor Affine
}

\author{
Fulvio Domini \\ Cognitive Technology Laboratory, AREA Science Park
}

\author{
Myron L. Braunstein \\ University of California, Irvine
}

\begin{abstract}
The relationship between simulated and judged depth separations for pairs of probe dots on planar surface patches was examined in a series of 6 experiments. The simulated slant of the patches was varied without varying the simulated depth separation of the probe dots by varying the depth gradient orthogonal to the direction determined by the probe dots on the image plane. Judged depth separation varied with mean slant for constant simulated depth separations. When observers judged depth separations along a closed path, the integral of the signed depths did not sum to zero, as would be required in Euclidean geometry. These results are inconsistent with the view that the mapping between simulated and perceived 3-D structure is affine and indicate that, in general, the perceived structure cannot be represented in either a Euclidean space or an affine space. Moreover, these results are consistent with a first-order temporal analysis of the optic flow.
\end{abstract}

A pattern of moving two-dimensional (2-D) features on a flat screen can give rise to a compelling impression of three-dimensionality. This phenomenon has been called the kinetic depth effect (Wallach \& O'Connell, 1953) or structure from motion (Ullman, 1979). Numerous attempts have been made to understand the underlying perceptual process and to answer the question of how the three-dimensional (3-D) properties of the perceived object are related to characteristics of the moving pattern. Much of the research on this topic has been influenced by the computational approach to vision (Marr, 1982) and has sought algorithms that could recover the real structure of the distal objects from moving patterns. Indeed, the main issue has been to find the minimal conditions and constraints that are sufficient for an ideal observer to recover the 3-D Euclidean structure of an object from 2-D moving images and to investigate the psychological validity of the theoretical findings (see Braunstein, Hoffman, Shapiro, Andersen, \& Bennett, 1987, for a discussion).

Recently, the view that the structure derived by the perceptual system has the same Euclidean properties as the

Fulvio Domini, Cognitive Technology Laboratory (a collaboration between the Department of Psychology of the University of Trieste and INSIEL SpA, a software company), AREA Science Park, Trieste, Italy; Myron L. Braunstein, Department of Psychology, University of California, Irvine.

This research was supported by National Science Foundation Grant SBR-9511198. The experiments were conducted at the University of California, Irvine, during an extended visit by Fulvio Domini. A partial report of these results was presented at the meeting of the Association for Research in Vision and Ophthalmology in Ft. Lauderdale, Florida in April 1996.

We thank A. Saidpour and J. Turner for helpful discussions and J. Lappin, J. Liter, and J. Todd for valuable comments on an earlier version of this article.

Correspondence concerning this article should be addressed to Fulvio Domini, Cognitive Technology Laboratory, AREA Science Park, Padriciano 99, Trieste, Italy. Electronic mail may be sent to fulvio@psicoiris.area.trieste.it. projected 3-D object has been questioned by a number of investigators. Empirical findings show that Euclidean quantities such as slant (Braunstein, Liter, \& Tittle, 1993; Domini, Caudek, \& Gerbino, 1995), angles (Todd \& Bressan, 1990), and depth (Caudek \& Proffitt, 1993) are misperceived by human observers. These findings are inconsistent with mathematical models that recover the 3-D Euclidean structure from moving images. Furthermore, evidence has been provided that two orthographic views of a moving object are sufficient for human observers to perceive a 3-D structure and that adding views does not substantially influence observers' performance in judgments about the structure (Liter, Braunstein, \& Hoffman, 1993; Todd, Akerstrom, Reichel, \& Hayes, 1988; Todd \& Bressan, 1990; Todd \& Norman, 1991). ${ }^{1}$ Two orthographic views of a rigid object are compatible with a one-parameter family of 3-D rigid interpretations (Bennett, Hoffman, Nicola, \& Prakash, 1989; Koenderink \& van Doorn, 1991; Todd \& Bressan, 1990). Because the perceptual system uses this mathematically ambiguous information to derive a 3-D structure, some researchers have pointed out that more general properties, rather than the specific 3-D Euclidean relationships, may be preserved in the percept. Moreover, it has been proposed that the derived object is related to the projected object by means of a linear scaling in the depth dimension (Todd \& Norman, 1991). A linear scaling preserves the affine properties of an object, such as the ordinal relationships and the parallelisms, but in general does not preserve Euclidean properties such as angles and distances (Koenderink \& van Doorn, 1991).

\section{Representational Space and Mapping}

Euclidean models as well as affine models that describe the perceptual derivation of 3-D structure from optic flow

\footnotetext{
${ }^{1}$ Adding views may influence performance if the amount of rotation (degrees of angular rotation) displayed increases with an increase in the number of views (Hildreth, Grzywacz, Adelson, \& Inada, 1990; Husain, Treue, \& Andersen, 1989).
} 
make very precise predictions about the relationships between the 3-D properties of the perceived object and the moving projections. Such models can be characterized by two important features: the representational space and the mapping between the 3-D object and this space.

The representational space of the Euclidean algorithms (Ullman, 1979) is the 3-D Euclidean space, and the mapping associates the projected 3-D features to elements of the perceived object by preserving the same Euclidean structure of the projected object. Because the sequence of 2-D images is inherently ambiguous with regard to 3-D structure, assumptions about the nature of the motion and the projection must be introduced in the mapping process. For example, it is possible to derive the 3-D Euclidean structure of an object from three views of four points if we assume rigid motion and orthographic projection (Ullman, 1979).

Koenderink and van Doorn (1991) proved that it is possible to derive the 3-D affine structure from two orthographic projections of four points. The constraint of rigidity is not necessary, but a 3-D affine transformation is assumed. The representational space of Koenderink and van Doorn's algorithm is a 3-D affine space, that is, a space where two objects that are linear transformations of each other are not discriminable. If the visual space is affine, therefore, only affine judgments are possible within this space. For example, it is possible to discriminate between parallel and nonparallel lines, compare segment lengths along parallel directions, and judge the coplanarity of points (Todd \& Bressan, 1990; Tittle, Todd, Perotti, \& Norman, 1995), but it is impossible to discriminate between 3-D structures that are related by linear stretching along the line of sight (Todd \& Norman, 1991) and to make accurate metric judgments of. such quantities as absolute length and angles (Todd \& Bressan, 1990). Moreover, Todd, Tittle, and Norman (1995) and Tittle et al. (1995) suggested that if the intrinsic structure of perceived space were Euclidean, whereas its extrinsic structure relative to the environment was not, then we should expect rotating objects to appear, in general, nonrigid.

In the current article we focus on the mapping as well as on the representational space that characterizes the structurefrom-motion process. ${ }^{2}$ We show, in general, (a) that the mapping between the simulated structure and the perceived object is not linear and therefore that the perceived and the simulated objects are not affinely related, (b) that depth judgments are internally inconsistent and therefore cannot be represented in Euclidean space, and (c) that direct comparisons of depth separations along the same direction are inaccurate, which indicates that the representational space is not affine.

Domini et al. (1995) recently suggested that perceived slant is a nonlinear function of a first-order temporal property of the optic flow, the deformation, and that the perceived tilt is accurately recovered. As shown below, and in Appendix A, this model predicts that the mapping between simulated depth and perceived depth will be nonlinear and that judgments of perceived depth will not be internally consistent.

\section{The Model}

The expectation that varying the slant of a surface patch will result in a nonlinear relationship between simulated and perceived depth is based on a model in which a heuristic procedure derives the slant of a planar surface that rotates in depth from two orthographic projections of the points on the surface. The orientation of a planar patch in 3-D space can be described in terms of its slant $(\sigma)$ and tilt $(\tau)$. Slant is defined as the angle between the line of sight (i.e., the $z$-axis) and the normal to the patch. This angle varies over a range of $90^{\circ}$, with slant equal to zero if the patch lies perpendicular to the line of sight (i.e., parallel to the $x-y$ plane). Tilt is defined as the angle between the projection of the normal to the patch and the $x$-axis.

Let us consider the optic flow produced by the orthographic projection of a patch having slant $\sigma$ and tilt $\tau$ and undergoing a generic 3-D rigid motion. The differential of the optic flow can be decomposed into four components (the differential invariants): curl, div, $\operatorname{def}_{1}$ and $\operatorname{def}_{2}$. The curl component describes a pure rigid rotation in the image plane; the div component describes an isotropic contraction and expansion; the $\operatorname{def}_{1}$ and $d e f_{2}$ components describe two orthogonal shears (Koenderink \& van Doorn, 1986; Todorovic, 1993). It is easy to show that the square root of the sum of the squared $d e f_{1}$ and $d e f_{2}$ is equal to the product of the slant (expressed as a tangent) of the planar patch $(\sigma)$ and its component of angular velocity $(\omega)$ parallel to the image plane. This quantity is the deformation (def; see also Domini, Caudek, \& Proffitt, 1997; Koenderink, 1986; Koenderink \& van Doorn, 1976, 1986):

$$
d e f=\sqrt{d e f_{1}^{2}+d e f_{2}^{2}}=\tan \sigma \omega .
$$

From the first-order optic flow (i.e., from two views), it is possible to derive, up to a reflection, the tilt of the surface and the component of angular velocity perpendicular to the image plane (Hoffman, 1982). However, the slant of the surface $(\sigma)$ and the component of angular velocity $(\omega)$ parallel to the image plane are undetermined. As can be seen in Equation 1, there are infinite pairs of $\sigma$ and $\omega$ that produce the same def. Domini et al. (1995) found that the perceived slant from multiple orthographic projections of a surface undergoing a 3-D rotation $\left(\sigma^{\prime}\right)$ is a monotonically increasing function of $d e f$ and that the tilt $\tau^{\prime}$ is correctly derived:

$$
\begin{gathered}
\boldsymbol{\sigma}^{\prime}=f(\text { def }) \\
\tau^{\prime}=\tau .
\end{gathered}
$$

\footnotetext{
${ }^{2}$ An extensive literature exists on the intrinsic geometry of the perceptual space for stereopsis (see Indow, 1991, for a review). Moreover, experiments have been presented assessing internal consistency of the metric judgments in shape from shading (Koenderik, van Doorn, \& Kappers, 1992) and full cues environments (F. N. Norman, Todd, Perotti, \& Tittle, 1996). This issue has been addressed only recently for structure-from-motion (SFM) displays by Werkhoven and van Veen (1995), who found that observers are inaccurate in making depth relief judgments.
} 


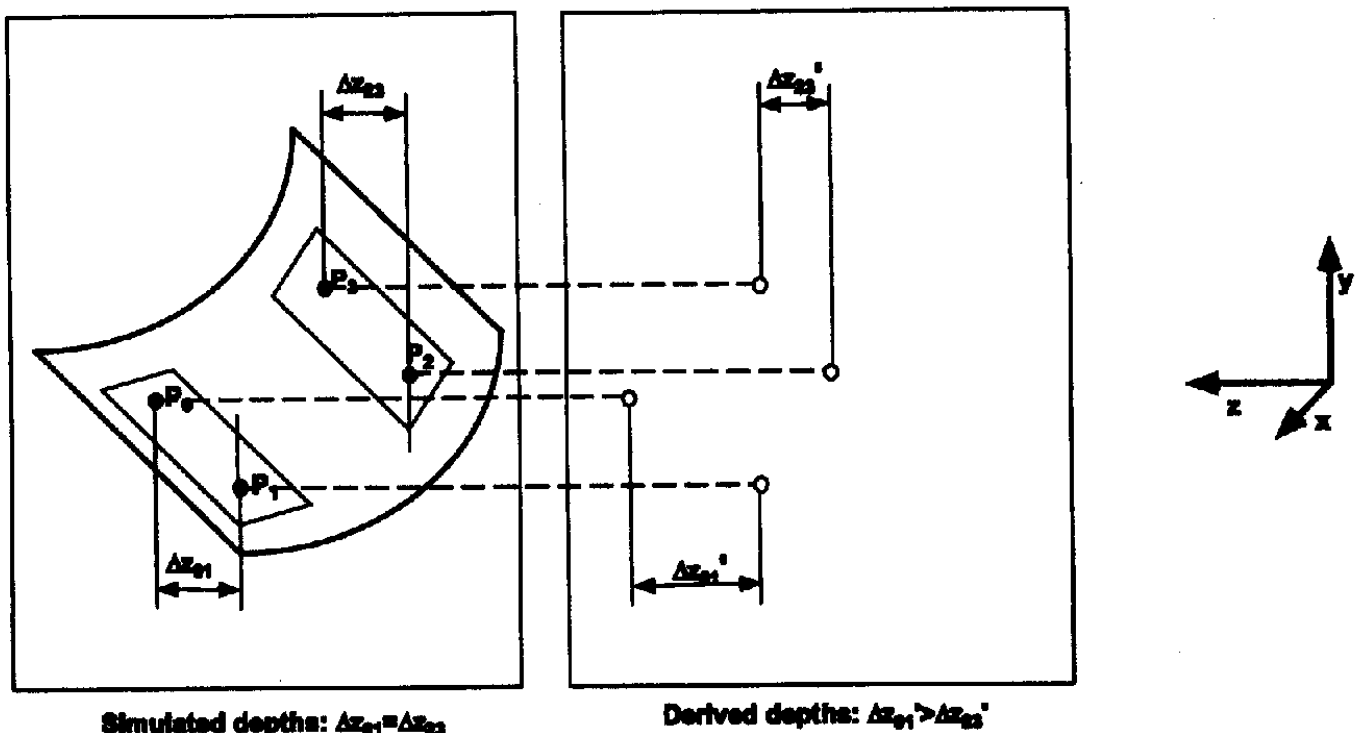

Figure 1. Prediction of the nonlinear model. The left panel shows a curved surface and two pairs of dots, $\mathbf{P}_{0}, \mathbf{P}_{1}$ and $\mathbf{P}_{2}, \mathbf{P}_{3}$, on two patches having different slants. The two pairs have the same simulated depth separations. The derived depth separations (right panel) are different, because the projected deformations produced by the two patches are different.

It can be shown (see Appendix $A$ for a derivation) that if Equations 2 and 3 are true, then the derived relative depth between two points on the surface is

$$
z^{\prime}=z \omega \frac{f(\operatorname{def})}{\operatorname{def}},
$$

where $z$ is the simulated relative depth between the two points. ${ }^{3}$ Equation 4 does not allow us to make a prediction about the function that relates the derived depth to def unless we make an assumption about the nature of the function $f(d e f)$. However, we can consider two categories of functions: (a) $f($ def) is proportional to def, or (b) $f(d e f)$ is not proportional to def. We call the first model affine and the second model nonlinear.

If the model is affine, then $f(\operatorname{def})=k^{*} \operatorname{def}$ and

$$
z^{\prime}=(k \omega) z
$$

Therefore, the derived depth is proportional to the simulated depth and does not depend on def.

If the model is nonlinear, then $f(\operatorname{def}) / d e f=F($ def $)$ is not constant, and

$$
z^{\prime}=[\omega F(\text { def })] z
$$

In this case the proportional factor between the derived and the simulated depth changes with def. Because

$$
z^{\prime}=[\omega F(\tan \sigma \omega)] z
$$

we can vary the expected $z^{\prime}$ for constant values of simulated $z$ by varying $\tan \sigma$ while maintaining a constant value of $\omega$.
The affine and nonlinear models describe very different processes of structure-from-motion derivation. To characterize these processes, let us consider a surface rigidly rotating about a generic axis. In Equations 5 and $6, \omega$ can be considered constant, because we simulated a constant angular velocity. The affine model derives a structure that is affinely related to the simulated one, because the derived depth separation of every pair of points is related to the simulated depth separation by a linear stretching in depth (Equation 5). On the other hand, the structure derived by the nonlinear model is, in general, not affinely related to the simulated structure. Moreover, it cannot be represented in Euclidean space. Let us consider the surface depicted in Figure 1. The left panel shows the simulated surface. The pairs of points $\mathbf{P}_{0}, \mathbf{P}_{\mathbf{1}}$ and $\mathbf{P}_{\mathbf{2}}, \mathbf{P}_{\mathbf{3}}$ have the same relative depth. However, they lie on patches that have different slants. Therefore, the values of def produced by the projection of the 3-D motion of the two patches are different, because they are the product of different slants and the same component of angular velocity $\omega$. It follows, in general, that the derived depth separations of the two pairs of points are different and, therefore, that the mapping is not linear (see Equation 6 and the right panel of Figure 1). On the other hand, the pairs of point $\mathbf{P}_{\mathbf{6}}, \mathbf{P}_{\mathbf{3}}$ and $\mathbf{P}_{\mathbf{1}}, \mathbf{P}_{\mathbf{2}}$ have the same relative depth and lie on patches that have the same slant. The derived depth separations of the two pairs are therefore the same. The algebraic sum of the derived depth separations along the closed path $\mathbf{P}_{\mathbf{0}}, \mathbf{P}_{\mathbf{1}}, \mathbf{P}_{\mathbf{2}}, \mathbf{P}_{\mathbf{3}}$ does not vanish, because $\mathbf{P}_{0} \mathbf{P}_{\mathbf{1}}^{\prime}-\mathbf{P}_{\mathbf{2}} \mathbf{P}_{\mathbf{3}}^{\prime}$ is different from zero and $\mathbf{P}_{0} \mathbf{P}_{\mathbf{3}}^{\prime}-\mathbf{P}_{\mathbf{1}} \mathbf{P}_{\mathbf{2}}^{\prime}$ is equal to 0 . This

\footnotetext{
${ }^{3}$ Equation 4 does not describe the process that derives a structure from moving images, because $z$ and $\omega$ are properties of the distal stimulus. Such a process is described in Appendix A.
} 
property is called internal inconsistency and is not a property of Euclidean space. Euclidean geometry is therefore inappropriate to describe the representational space of a nonlinear mapping.

The experiments described in the present article were designed to investigate the effect of the slant of surface patches passing through pairs of probe dots on the perception of the depth separation (i.e., the distance between the two dots along the line of sight) of the dots in each pair. The nonlinear model predicts a nonlinear relationship between simulated depth and perceived depth when slant is varied. ${ }^{4}$ In the first five experiments the observers matched the perceived depth separation of two probe dots with the length of a line that appeared on a separate monitor. In the first, second, and third experiments, the two probe dots were positioned on a planar surface, at the intersection of two planar surfaces, and in a cloud of dots, respectively. It is important to note that if the nonlinear model predicts the results of the first three experiments, an affine mapping is not necessarily ruled out. We could speculate that the slant of the surfaces on which the probe dots are located leads to different scale factors for the affine mapping for different displays. This issue was directly addressed in the fourth and fifth experiments, in which the two probe dots were positioned in different regions of a single curved or planar surface. It can be argued, however, that the method of adjusting a line to make absolute depth judgments could be inappropriate for investigating whether or not the intrinsic structure of the perceived space is affine, because this particular task might require the observers to mentally rotate the perceived depth. Therefore, we used a different method in the sixth experiment in which observers directly compared the depth separations for two pairs of probe dots positioned on two planar surfaces rotating rigidly about the same axis. The observer's task was to adjust the simulated distance in depth between the dots in one pair so that it matched the perceived separation in depth of the dots in the other pair.

\section{Experiment 1: A Single Surface}

The affine model predicts that the perceived depth separation of two probe dots will be proportional to the simulated depth separation (see Equation 5). The nonlinear model predicts that the derived depth separation will also be a function of the slant of the planar surface that passes through the dots (when simulated slant is varied independently of the simulated depth separation of the probe dots; see Equation 7). The purpose of the first experiment was to investigate which of these two models predicts human performance.

We simulated an orthographic projection of a planar surface rigidly rotating about the vertical axis with two identifiable points positioned on the surface. The observer's task was to adjust a line that appeared on a separate monitor in order to match the perceived depth separation of the two points.

\section{Method}

Observers. The observers were 6 graduate students at the University of California, Irvine. They were paid for their participation and were naive to the purposes of the experiments. Only 2 of them were familiar with structure-from-motion (SFM) displays. All had normal or corrected-to-normal vision (20/40 on the Snellen eye chart).

Design. Three independent variables were examined: the simulated distance between the two probe dots along the line of sight $(9.98 \mathrm{~cm}$ or $19.96 \mathrm{~cm})$, the slant of the planar surface that passed through the two probe dots $(0.5,0.81,1.24$, or 2.0 for the depth separation of $9.98 \mathrm{~cm} ; 1,1.44,2.21$, or 4.0 for the depth separation of $19.96 \mathrm{~cm}$ ), and the initial direction of 3-D rotation (to the right or to the left). (Unless otherwise specified, slant values reported here are the tangents of the slant angle $\sigma$.) All of the variables were run within observers.

Apparatus. The stimulus displays were presented on a Xytron 19-in. (48-cm) color display scope with a Tucker-Davis six-channel digital-to-analog interface controlled by a Dell Pentium 90 computer. Dots were displayed at a rate of $60 \mathrm{frames} / \mathrm{s}$. Plotting accuracy was 16 bits in $X$ and $Y$. The monitor was viewed monocularly through a viewing tube from a distance of approximately $200 \mathrm{~cm}$. The viewing tube limited the visible portion to a circular region $27.9 \mathrm{~cm}$ in diameter $\left(8^{\circ}\right.$ of visual angle). The response display was presented on a separate 14 -in. $(36-\mathrm{cm})$ monitor to the observer's right. The response device consisted of a joystick that the observer could use to adjust a line on the response display from a minimum length of $0 \mathrm{~cm}$ to a maximum length of $27.5 \mathrm{~cm}$. The direction of the line on the response display was parallel to the line of sight for the stimulus display.

Stimuli. The displays were composed of light red dots on a black background. The two probe dots were light green dots. For each display, 100 dots were positioned randomly in a circular region $27.9 \mathrm{~cm}$ in diameter. The motion of the dots simulated the orthographic projection of points rotating rigidly in 3-D space about the vertical axis through $\pm 6^{\circ}$. The simulated surface extended beyond the visible region of the screen so that the bounding contours did not become visible during the rotation. One entire cycle of rotation took $2 \mathrm{~s}$. Figure 2 shows the simulated structure (left panel) and the projection on the image plane (right panel). The two probe dots $\mathbf{P}_{0}$ and $\mathbf{P}_{1}$ were separated vertically by $19.96 \mathrm{~cm}(\Delta y)$. The simulated depth separation of the probe dots was either $9.98 \mathrm{~cm}$ or $19.96 \mathrm{~cm}(\Delta z)$. The simulated depth separation varied by only $0.6 \%$ of the maximum value during the rotation. The axis of rotation was $19.96 \mathrm{~cm}$ from the midpoint of an imaginary segment connecting the two dots and was placed behind them to minimize the likelihood of depth reversals (Braunstein et al., 1993). When the depth separation was $19.96 \mathrm{~cm}$, points $\mathbf{P}_{0}$ and $\mathbf{P}_{1}$ projected maximum displacements of $0.60^{\circ}$ and $1.79^{\circ}$ of visual angle, respectively, corresponding to mean velocities on the image plane of $0.60 \%$ and $1.79 \%$. When the depth separation was 9.98 $\mathrm{cm}$, the projected maximum displacements were $0.90^{\circ}$ and $1.50^{\circ}$ of visual angle, corresponding to mean velocities of $0.90 \%$ and $1.50 \%$ s. For each depth separation, four different slanted planar surfaces passing through the two probe dots were simulated. Let us indicate with $\sigma_{\min }$ the minimum value of slant that a planar surface passing through two probe dots can take (see the left panel of Figure 2). We selected a second slant, $\sigma_{\max }$, such that $\tan \sigma_{\max }=$ $4 \tan \sigma_{\text {min }}$. On the basis of pilot studies, we divided the interval

\footnotetext{
${ }^{4}$ The present experiments are not intended as a general test of the nonlinear model; only the relationship between simulated slant and judged depth is examined.
} 


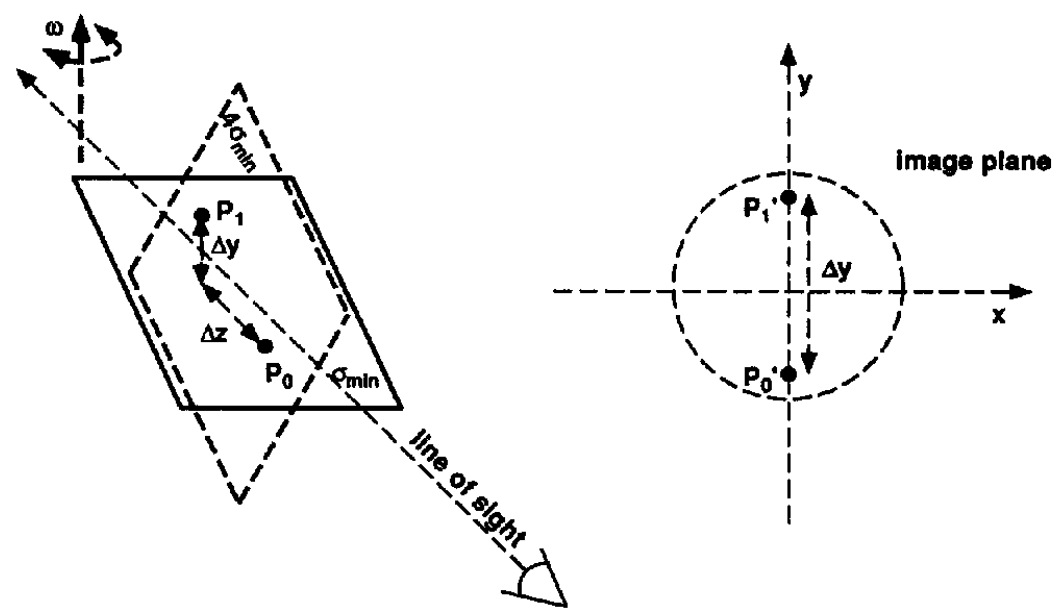

Figure 2. The simulated structure in Experiment 1 (left panel) and the projection on the image plane (right panel). The two probe dots $P_{0}$ and $P_{1}$ were separated vertically by $\Delta y$ and along the line of sight by $\Delta z$. The axis of rotation was behind the probe dots ( $\omega$ indicates the simulated angular velocity). $\sigma_{\min }$ represents the minimum value of slant that a planar surface passing through two probe dots can take. The surface that has the minimum value of slant is depicted with a filled line. We selected a maximum slant, $\sigma_{\max }$, such that $\tan \sigma_{\max }=4 \tan \sigma_{\min }$. The dashed line represents the surface with slant $\sigma_{\max }$.

between $\sigma_{\min }$ and $\sigma_{\max }$ into three equal-angle (rather than equaltangent) intervals. For the depth separation of $9.98 \mathrm{~cm}$, this resulted in slant angles of $26.6^{\circ}, 39.0^{\circ}, 51.1^{\circ}$, and $63.4^{\circ}$ (tangents of 0.5 , $0.81,1.24$, and 2.0). For the depth separation of $19.96 \mathrm{~cm}$, the slant angles were $45.0^{\circ}, 55.3^{\circ}, 65.6^{\circ}$, and $76.0^{\circ}$ (tangents of $1.0,1.44$, 2.21 , and 4.0 ).

A generic planar surface can be described by the two components of depth gradient along the $x$ and $y$ axes: $g_{1}$ and $g_{2}$ (see Appendix A). The vertical component $\left(g_{2}\right)$ was the same for every surface that passed through the simulated probe dots and was calculated as the ratio between the depth separation and the vertical displacement. The value of this component was 1 for the $19.96-\mathrm{cm}$ depth separation and 0.5 for the 9.98 -cm depth separation. Because slant is defined as the square root of the sum of the squares of the two components of the depth gradient, the generic surface passing through the two probe dots took the minimum value of slant when the horizontal component of the depth gradient $\left(g_{1}\right)$ was null. Therefore, the slant was increased by increasing the horizontal component of the depth gradient of the planar surfaces. The horizontal component of the depth gradient was $0,1.04,1.97$, or 3.87 for the $19.96-\mathrm{cm}$ depth separation and $0,0.64,1.13$, or 1.94 for the 9.98-cm depth separation.

Procedure. The observers were instructed to judge the depth separation of the two green dots on the simulated planar surface. They were told that depth separation means the distance between the two dots along the line of sight. A drawing was used to illustrate this concept. They were instructed to use a joystick to adjust the length of a white line that appeared on the computer screen positioned to their right in order to match the perceived relative depth. They were told that the direction of the depicted line was exactly the direction along which they had to judge the relative depth between the two green dots. When they were satisfied with the length of the comparison line, they pressed the trigger of the joystick to initiate the next trial. The responses were not timed. The observers participated individually in two sessions of 48 trials each, presented in random order. A training session of 16 trials preceded the actual experiment.

\section{Results and Discussion}

A 2 (session) $\times 2$ (depth) $\times 4$ (slant) $\times 2$ (rotation) within-subjects analysis of variance (ANOVA) was performed on the judged depths. There were significant effects of depth, $F(1,15)=9.79, p<.05$, and slant, $F(3,15)=$ $8.29, p<.01$, and a significant interaction, $F(3,15)=5.92$, $p<.01$. No other main effects or interactions reached significance.

Mean judged depth for each level of simulated depth and slant is shown in Figure 3. Figure 4 shows the plots for individual observers. Five observers showed the same trends; only Observer T.L. showed a nonmonotonic relationship between simulated slant and judged depth. This observer reported in the debriefing session that he perceived

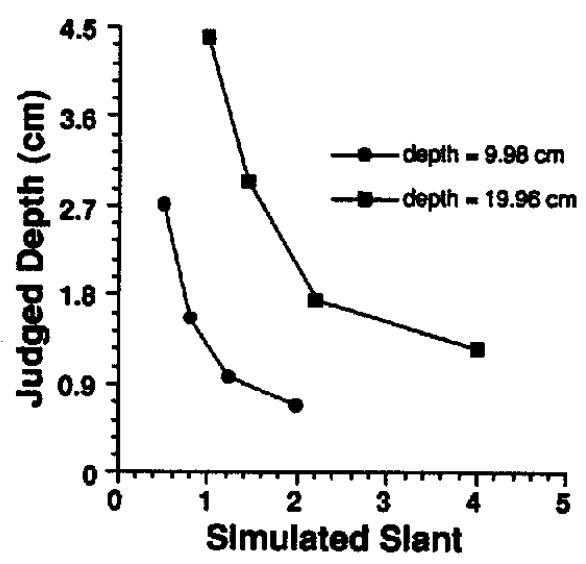

Figure 3. Mean judged depth for each level of simulated depth as a function of the simulated slant in Experiment 1. 

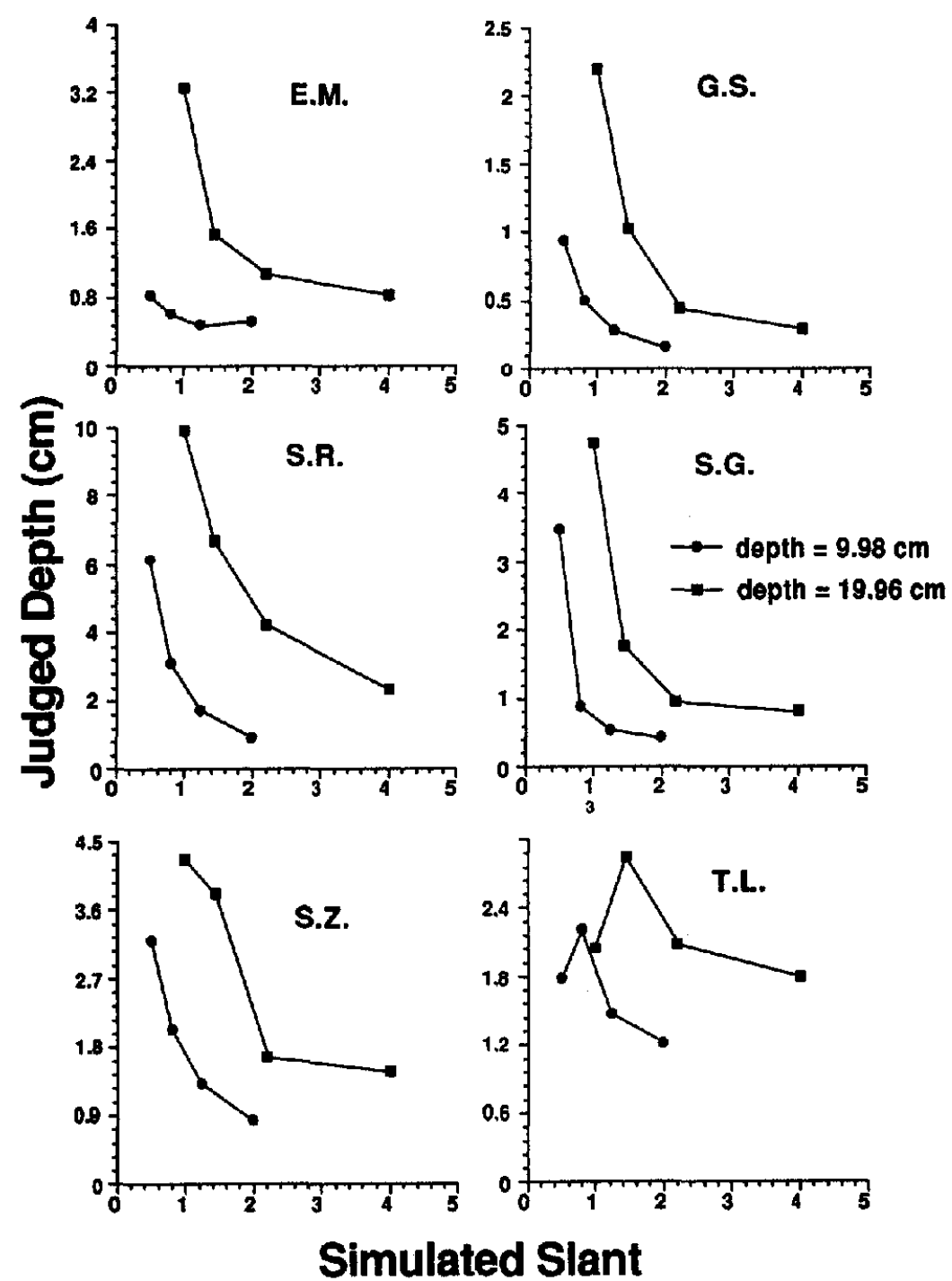

Figure 4. Mean judged depth for each level of simulated depth as a function of the simulated slant for individual observers in Experiment 1.

the least slanted surfaces for both simulated depth separations as undergoing a nonrigid horizontal stretching in the image plane. Though the observers showed similar trends, the depth scalings were very different. These differences may be attributable to differences in the perceived distance of the object from the observer. Observers reported perceived distances ranging from $30 \mathrm{~cm}$ to $200 \mathrm{~cm}$, and these were in general related to observers' average depth judgments.

In an additional analysis we calculated for each observer the "reliability" of the judgments in each condition expressed as the standard deviation of the adjustments relative to the mean (F. N. Norman et al., 1996). A 2 (depth) $\times 4$ (slant) within-subjects ANOVA was performed on the reliabilities. There was a significant effect of depth, $F(1,15)=$ $52.092, p<.01$, but not of slant. The "reliability" was $42 \%$ for the smaller simulated depth and $30 \%$ for the larger simulated depth. These values, however, should be compared with the magnitude of the effect of the slant variable. For a simulated depth of $9.98 \mathrm{~cm}$, the mean perceived depth for the minimum slant was $401 \%$ of the mean perceived depth for the maximum slant; for a simulated depth of $19.96 \mathrm{~cm}$, the mean perceived depth for the minimum slant was $350 \%$ of the mean perceived depth for the maximum slant. Appendix B presents the "reliability" measures averaged among observers in each condition of Experiments 1 through 6.

There are two important aspects in the present results: (a) The slant of the simulated planar surface that passed through the two probe dots influenced the judgments of the observers. The mean judged depth decreased as the simulated slant increased. It dropped to $30 \%$ of the maximum judged depth for the simulated depth of $19.96 \mathrm{~cm}$ and to $25 \%$ of the maximum value for the simulated depth of $9.98 \mathrm{~cm}$. The different ratios between the maximum and minimum values for the two simulated depth separations explain the signifi- 
cant interaction between slant and simulated depth separation in determining judged depth separation. (b) Depending on the relative surface slants, the judged depth separation of the probe dots could be greater for the smaller simulated depth separation than for the larger simulated depth separation (see Figure 3). This result is especially surprising if we consider that one simulated depth separation was twice the other one.

Our motivation for the present experiment was to test the validity of two mutually exclusive models that derive the depth separation of two probe dots from the first-order temporal properties of the optic flow. These results suggest that the affine model has to be rejected, because the judged depth separation of two probe dots is influenced not only by the simulated depth separation but also by the slant of the planar surface that passes through the points. When the same depth separation was simulated, the judged depth separation was a monotonically decreasing function of the simulated slant.

\section{Experiment 2: Two Surfaces}

In Experiment 2 we investigated the effect of the simulated slants of two transparent planar surfaces passing through the probe dots on the judged depth separation of the dots. Our purpose was to determine whether judged depth can be influenced by the slant of more than one surface and, if so, whether the judgments are related to the average slant of the surfaces.

\section{Method}

Observers. Five of the observers who participated in Experiment 1 participated in this experiment.

Design. Two independent variables were examined: the slants of the surfaces passing through the probe dots and the initial direction of 3-D rotation (to the right or to the left). The slant variable had six levels: Either a single surface passed through the probe dots with slants of 1.0,1.44, or 2.21, or two surfaces passed through the probe dots with slants of 1.0 and $1.44,1.0$ and 2.21 , or 1.44 and 2.21. The first three conditions were equivalent to the single-surface conditions in Experiment 1. All of the variables were run within observers.

Apparatus. The apparatus was the same as that in Experiment 1.

Stimuli. The displays were similar to those in Experiment 1 except that 200 dots were positioned randomly in the circular region and the two probe dots were always separated vertically by $19.96 \mathrm{~cm}$. The simulated depth separation of the two probe dots was also $19.96 \mathrm{~cm}$, and the axis of rotation was $19.96 \mathrm{~cm}$ from the midpoint of an imaginary segment connecting the two dots. We used the three least slanted surfaces from the greater depth separation condition of Experiment 1. The slants expressed as angles were $45.0^{\circ}, 55.3^{\circ}$, or $65.6^{\circ}$, with corresponding tangents of $1.0,1.44$, or 2.21 . In the three single-slant conditions, 200 dots were assigned to one surface, as in Experiment 1. In the three two-slant conditions, 100 dots were assigned randomly to each surface.

Procedure. The procedure was the same as that in Experiment 1 except that the observers participated individually in two sessions of 60 trials presented in random order, and a training session of 12 trials preceded the actual experiment. Furthermore, observers were told that two transparent surfaces would be simulated on half of the trials.

\section{Results and Discussion}

A 2 (session) $\times 6$ (surface slants) $\times 2$ (rotation direction) within-subjects ANOVA was performed on the judged depths. There was a significant effect of surface slant condition, $F(5,20)=23.9, p<.001$, and of rotation direction, $F(1,4)=12.6, p<.05$. None of the other factors or interactions reached significance. In an additional analysis we calculated the "reliability" of the judgments in each condition of Experiment 2 (see Appendix B).

Mean judged depth is plotted in Figure 5 as a function of the mean slant of the surfaces passing through the probe dots. (For the single-surface conditions the mean slant is the slant of that surface.) When one surface was simulated, judged depth was a decreasing function of slant. When two surfaces were simulated, judged depth was a decreasing function of the mean slant.

The results of Experiment 1 indicated that judged depth separation of two points is influenced by the slant of the planar surface passing through the points. These results can be predicted by the nonlinear model, which derives the depth separation as a function of the slant of a surface that passes through the points. In Experiment 1 this function was a monotonically decreasing function of the slant. The results of Experiment 2 indicate that the perceived depth is a monotonically decreasing function of the mean slant of the planar surfaces that pass through the probe dots.

\section{Experiment 3: A Cloud of Dots}

The results of Experiment 2 indicated that judged depth separation of two probe dots is a decreasing function of the mean slant of the planar surfaces passing through the dots. Our purpose in Experiment 3 was to determine whether placing the probe dots on clearly defined smooth surfaces was required to obtain an effect of simulated slant on judged depth. In this experiment we tested the influence of mean

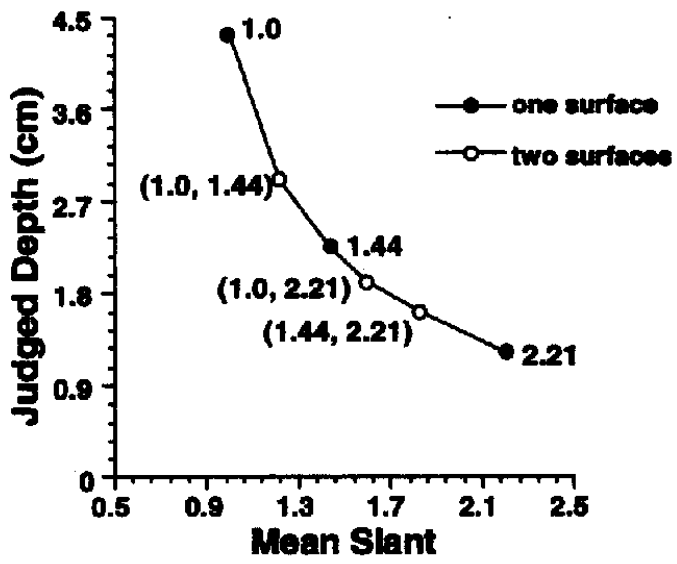

Figure 5. Mean judged depth for two structures (two surfaces and one surface) as a function of the mean slant in Experiment 2. 
slant on judged depth separation for dots randomly positioned in a region of 3-D space.

\section{Method}

Observers. The same 5 observers who participated in Experiment 2 participated in this experiment.

Design. Three independent variables were examined: the possible combinations of three planar surfaces (slants of 1.0 and 1.44 , 1.0 and 2.21, and 1.44 and 2.21 ), the structure (two surfaces vs. a cloud), and the initial direction of 3-D rotation (to the right or to the left). All of the variables were run within observers.

Apparatus. The apparatus was the same as that in Experiment 1. Stimuli. The stimuli in the two-surface conditions were the same as those in Experiment 2. In the "cloud" condition we replaced the two surfaces with a cloud of dots randomly distributed in the region delimited by the two surfaces. The procedure we used to generate the cloud of dots was to place every dot on a different planar surface passing through the probe dots. The slants of these planar surfaces were randomly selected from a uniform distribution (in degrees) over a range defined by the slant values of the two delimiting surfaces.

Procedure. The procedure was the same as that in Experiment 2 except that the observers were told that on half of the trials a cloud of random dots was simulated.

\section{Results and Discussion}

A 3 (surface combination) $\times 2$ (cloud vs. surfaces) $\times 2$ (rotation direction) within-subjects ANOVA was performed on the judged depths. There was a significant effect of surface combination, $F(2,8)=19.67, p<.01$, and of the structure (cloud vs. surfaces), $F(1,4)=25.58, p<.01$. None of the other factors or interactions reached significance. In an additional analysis we calculated the "reliability" of the judgments in each condition of Experiment 3 (see Appendix B).

Mean judged depths for each surface combination and for the two structures (surfaces and cloud) are plotted in Figure 6 as a function of the mean slant of the surfaces passing through the probe dots. (The mean slant of the two surfaces delimiting the cloud of dots was used in the cloud condition.) In the surfaces condition we replicated the results of Experiment 2. In the cloud condition the combinations of slants of the two surfaces delimiting the random dot region also influenced the judged depth separation of the probe dots. Furthermore, the mean perceived depth separation was greater for the cloud condition than for the surfaces condition. Let us consider, however, the plot of Figure 6. The mean judged depth separations in the cloud condition are plotted as a function of the mean slant of the surfaces delimiting the region of the 3-D random dots. This is an arbitrary choice, however. Alternatively, we could consider an average measure of the slants of the planar surfaces passing through the probe dots and each of the randomly generated dots in the cloud. In Figure 7 we replotted the mean judged depths in the cloud condition as a function of the mean slant of the planar surfaces passing through the two probe dots and each dot in the cloud. When plotted in this way, judged depth is a monotonically decreasing function of the mean slant.

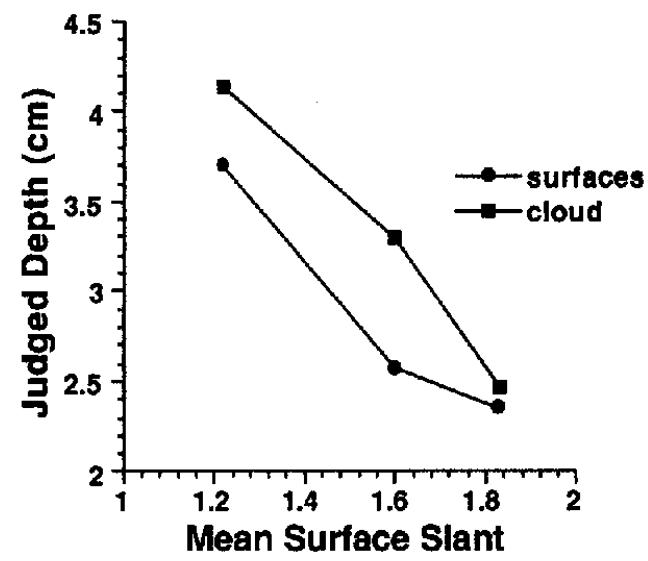

Figure 6. Mean judged depths for two structures (surfaces and cloud) as a function of the mean slant in Experiment 3. The mean slant of the two surfaces delimiting the cloud of dots was used in the cloud condition.

The results of Experiment 3 suggest that the judged depth separation between two probe dots is influenced by the average slant of the surfaces that pass through the points. These results also indicate that the effects of simulated slant on judged depth are not limited to displays in which the points are located on smooth surfaces.

\section{Experiment 4: Consistency of Depth Judgments}

The results of the three experiments previously described indicate that the slant or the pattern of slants of the planar surfaces that pass through two probe dots influences their perceived depth separation. The judged depth separation is, in general, a decreasing function of the mean of the slants of planar surfaces passing through the two probe dots and each additional dot in the display. Our purpose in Experiment 4

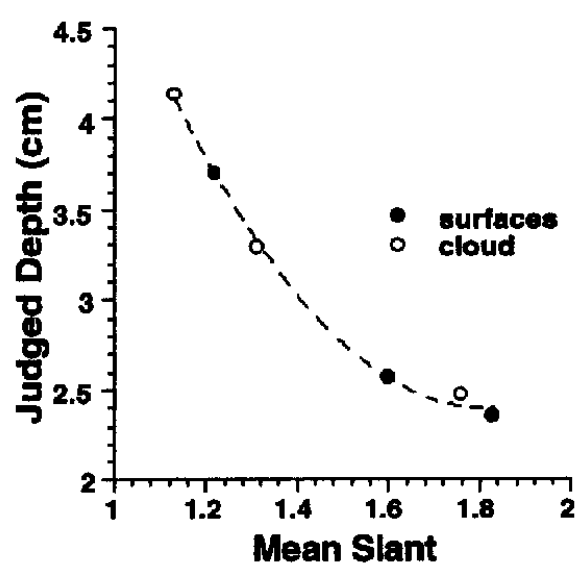

Figure 7. Mean judged depths for two structures (surfaces and cloud) as a function of the mean slant in Experiment 3. The mean slant of the planar surfaces passing through each dot and the two probe dots was used in the cloud condition. 
was twofold. First, it was to extend the previous findings to the general case of two probe dots lying on different patches of the same curved surface. Second, it was to study the internal consistency of metric judgments. Consider the left panel of Figure 8. It shows a curved surface and two pairs of probe dots lying on different regions of this surface. The two pairs of dots have the same depth separation but lie on two patches that have different slants. The pair $\mathbf{P}_{\mathbf{0}}, \mathbf{P}_{\mathbf{1}}$ in the figure lies on a patch having a slant $\sigma_{0,1}$ that is smaller than the slant $\sigma_{2,3}$ of the patch that contain's the pair $\mathbf{P}_{2}, \mathbf{P}_{3}$. If the results of the previous experiments are replicated, we would expect the perceived depth separations of the two pairs of dots to be different. Furthermore, we would expect the perceived depth separations of the pairs $\mathbf{P}_{0}, \mathbf{P}_{3}$ and $\mathbf{P}_{1}, \mathbf{P}_{2}$ to be the same, because both pairs lie on regions of the curved surface with identical slants. We should expect, therefore, that the sum $Z_{0,1}^{\prime}+Z_{1,2}^{\prime}$ is not equal to $Z_{2,3}^{\prime}+Z_{3,0}^{\prime}$, because, in this example, $Z_{0,1}^{\prime}>Z_{2,3}^{\prime}$ and $Z_{1,2}^{\prime}=Z_{3,0}^{\prime}$. If this happened, the judgments would be internally inconsistent because different paths of integration would give different results and the algebraic sum of the judgments along a closed path would not be zero.

\section{Method}

Observers. The 5 observers who participated in Experiments 2 and 3 participated in this experiment.

Design. Three independent variables were examined: shape (nine curved surfaces), probe pair positions (four positions), and the position of the axis of rotation (behind or in front of the simulated surface). All of the variables were run within observers.

Apparatus. The apparatus was the same as that in Experiment 1.

Stimuli. The displays were similar to those in Experiments 2 and 3 except that a single smoothly curved surface was simulated. The horizontal diameter of the projected surface was divided into three equal regions (see Figure 8). The two lateral regions simulated planar patches. These were connected by a central cylindrical patch. Each of the lateral regions had a slant value of 1.0, 1.44, or 2.21. As in Experiments 2 and 3, the vertical component of the depth gradient for each of these regions was 1 , and different values of slant were obtained by changing the value of the horizontal component. The depth gradient of the cylindrical patch was 1 . The horizontal gradient of the cylindrical patch varied smoothly between the two values of the horizontal gradient of the two lateral planar patches. The whole surface was, therefore, a smooth cylindrical surface (see the left panel of Figure 8). The intersection of the simulated surface with a generic horizontal plane was a smooth curve that had exactly the same shape for every vertical position of the horizontal plane. Figure 9 shows the nine surfaces obtained by combining the three possible slants on the left region with the three possible slants on the right region.

On half of the trials, the axis of rotation was behind the surface at a distance of $14.0 \mathrm{~cm}$ from the center, and on half of the trials it was in front at the same distance from the center. We manipulated the position of the axis of rotation in order to control the mean projected velocities of the probe dots positioned on the lateral surfaces. For the asymmetric surfaces rotating in front of the axis of rotation, for example, the mean projected velocity of the probe dots lying on the less slanted lateral surface was smaller than the mean projected velocity of the probe dots lying on the more slanted lateral surface.

The right panel of Figure 8 shows the projection on the image plane of the probe dots at the midpoint of the rotation cycle. The vertical separation of probe dot pairs $P_{0}, P_{1}$ and $P_{2}, P_{3}$ was $9.3 \mathrm{~cm}$, and the horizontal separation of the probe dot pairs $\mathbf{P}_{0}, \mathbf{P}_{\mathbf{3}}$ and $\mathbf{P}_{\mathbf{1}}, \mathbf{P}_{\mathbf{2}}$ was $18.7 \mathrm{~cm}$. Only two probe dots were shown on each trial. The simulated depth separations of the pairs of probe dots $\mathbf{P}_{\mathbf{0}}, \mathbf{P}_{\mathbf{1}}$ and $P_{2}, P_{3}$ were the same in all the displays and corresponded to $9.3 \mathrm{~cm}$. The depth separations of the pairs $\mathbf{P}_{0}, \mathbf{P}_{3}$ and $\mathbf{P}_{1}, \mathbf{P}_{\mathbf{2}}$ depended on the simulated slants of the lateral surfaces. When the lateral surfaces had the same slant (see the top panel of Figure 9), their depth separation was null. When the lateral surfaces were different, the
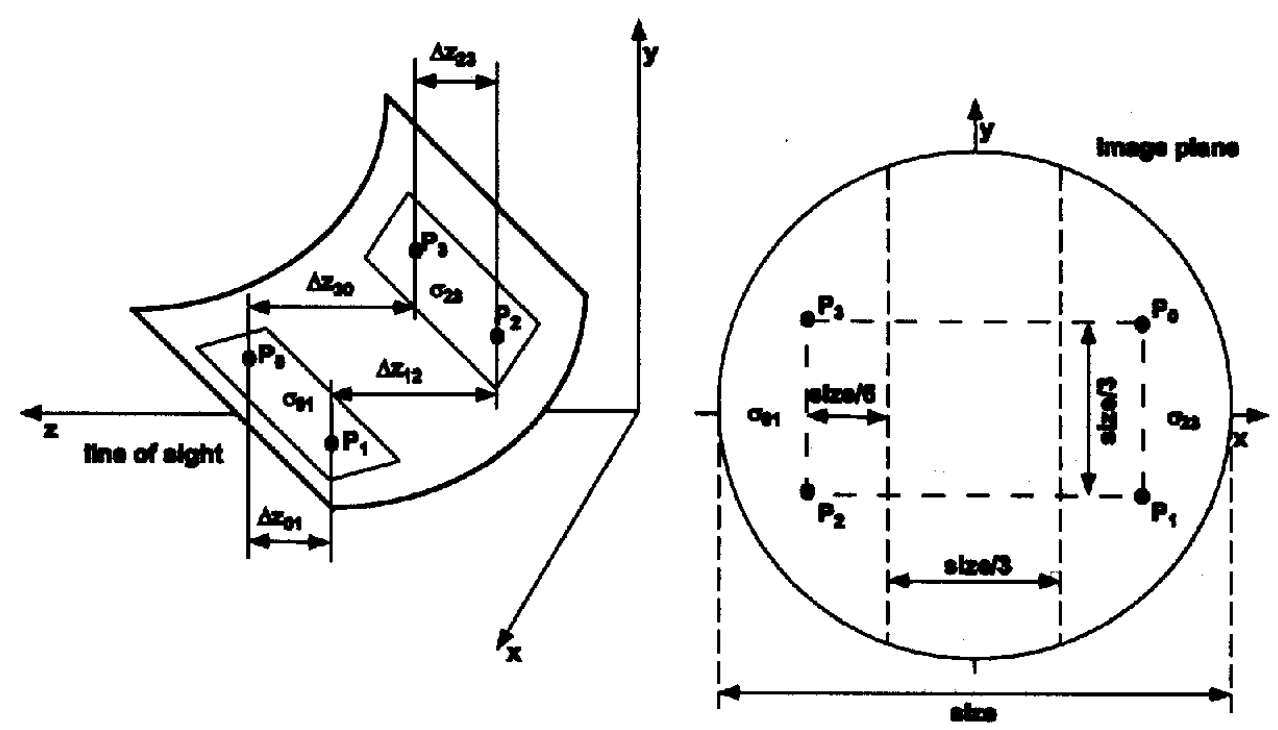

Figure 8. The simulated cylindrical structure in Experiment 4 (left panel) and the projection on the image plane (right panel). $\sigma_{0,1}$ and $\sigma_{2,3}$ indicate the slants of the two lateral patches; $\Delta z_{i j}$ indicates the simulated depth separation for each possible pair of probe dots $\left(\mathbf{P}_{\mathbf{i}}, \mathbf{P}_{\mathbf{j}}\right)$; and size indicates the diameter of the visible circular region of the stimulus display. 


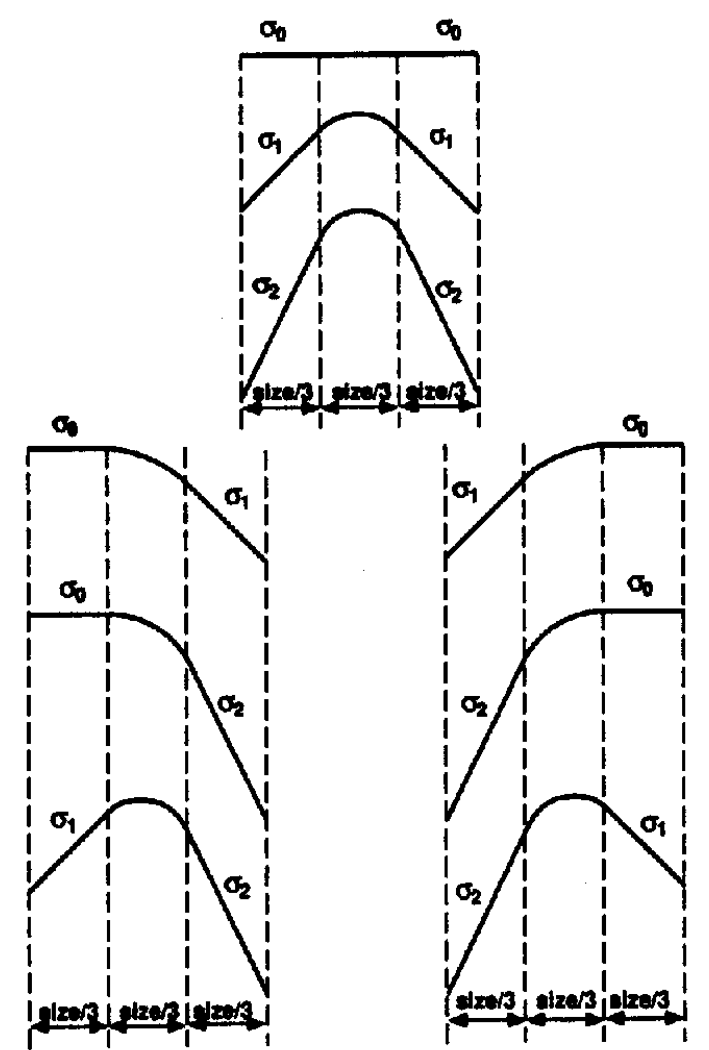

Figure 9. The intersections with a generic horizontal plane of the simulated cylindrical structures in Experiment 4. $\sigma_{0}, \sigma_{1}$, and $\sigma_{2}$ indicate the possible slants of the two lateral patches; size indicates the diameter of the visible circular region of the stimulus display.

depth separations were $14.9,8.8$, and $5.7 \mathrm{~cm}$ for pairs of surfaces with slants of 1.0 and $2.21,1.0$ and 1.44 , and 1.44 and 2.21, respectively.

Procedure. The procedure was the same as that in the previous experiments except that the observers were told that the pair of probe dots could appear in four different positions on the simulated smoothly curved surfaces in the stimulus display. Furthermore, they were asked to judge the depth separation of the probe dots at the midpoint of the rotation cycle. The observers participated individually in four sessions of 72 trials presented in random order.

\section{Results and Discussion}

Our first goal in Experiment 4 was to investigate the effect of the slant of a local planar patch of a smoothly curved surface on the judged depth separation of two probe dots lying on the patch. Therefore, a separate analysis of the depth judgments of the probe pairs $\mathbf{P}_{\mathbf{0}}, \mathbf{P}_{\mathbf{1}}$ and $\mathbf{P}_{\mathbf{2}}, \mathbf{P}_{\mathbf{3}}$ was performed. $\mathbf{A} 3$ (slant) $\times 2$ (probes $\mathbf{P}_{0}, \mathbf{P}_{\mathbf{1}}$ or probes $\mathbf{P}_{2}, \mathbf{P}_{\mathbf{3}}$ ) $\times$ 2 (axis position) within-subjects ANOVA was performed on the judged depths. There was a significant effect of slant, $F(2,8)=4.599, p<.05$. None of the other factors or interactions reached significance. In an additional analysis we calculated the "reliability" of the judgments in each condition of Experiment 4 (see Appendix B).

Mean judged depths for each simulated slant and for the two simulated axes of rotation are plotted in Figure 10 as a function of the slant of the surface passing through the probe dots. Though the interaction of the slant with the axis of rotation did not reach significance, $F(2,8)=2.97$, the plot suggests that the effect of surface slant was mainly due to the axis-behind conditions. When the axis of rotation was behind the surface, the judged depth decreased as the slant of the patch on which the probe dots were located increased. However, this effect was substantially reduced when the axis of rotation was in front. We chose to manipulate the position of the axis of rotation in order to control the mean velocity of the probe dots, which had been held constant in Experiments 1,2 , and 3 . The difference between the maximum and the minimum velocity of the probe dots $\mathbf{P}_{0}, \mathbf{P}_{1}$ and $\mathbf{P}_{2}, \mathbf{P}_{\mathbf{3}}$ was the same for each simulated surface. Therefore, the mean velocity covaried with the ratio between the maximum and the minimum velocity - the greater the mean velocity, the smaller the ratio. We calculated for each simulated slant the mean of the ratios between the maximum and the minimum velocities of the probe dots. Figure 11 shows the mean ratios as a function of the slant and the axis position. As we can see, the mean ratio increases when the slant increases if the axis of rotation is behind the surface, and the mean ratio decreases when the slant increases if the axis of rotation is in front. Let us assume that the ratio between the maximum and minimum velocities of the probe dots influenced the perceived depth separation. The positive correlation between ratio and judged depth separation when the axis of rotation was behind the surface would produce an effect of ratio in the same direction as that of the effect of slant in that condition. The negative correlation when the axis was in front of the surface would produce an effect of ratio in a direction opposite to that of the effect of slant in that condition. This would explain the reduced effect of slant when the axis of rotation was in front of the simulated surfaces. The relationship between ratio and judged depth separation was examined directly in Experiment 5 .

Our second goal in Experiment 4 was to investigate the ability of human observers to make metric judgments that

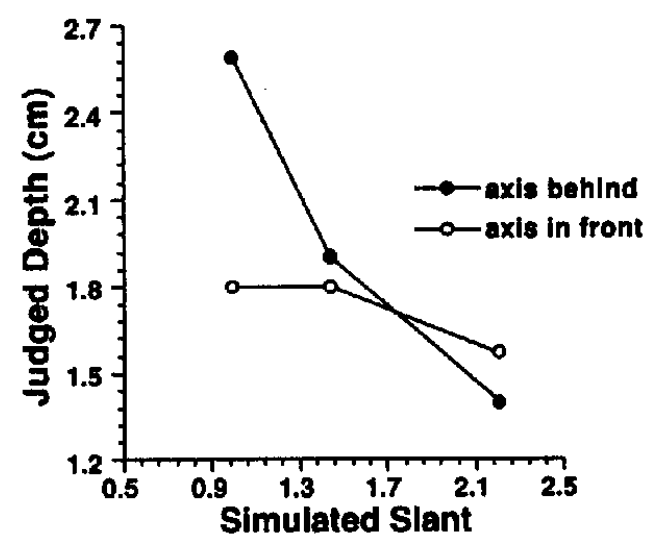

Figure 10. Mean judged depth for the two simulated axes of rotation as a function of the slant of the lateral patch on which the pair of probe dots was located in Experiment 4. 
are internally consistent. Observers, on separate trials, judged depth separations for each of the four probe dot locations, for each combination of surface and axis position. This provided a set of four depth judgments along a closed path (see the right panel of Figure 8) for each of the nine simulated surfaces and the two positions of the axis of rotation. We collected a complete set of these judgments in each of the four sessions. We calculated the integral, $I^{\prime}$, of the judged depth separation for each observer and each session for each of the 18 combinations of surface and axis position, where $I^{\prime}=Z_{0,1}^{\prime}-Z_{2,3}^{\prime}+Z_{3,0}^{\prime}-Z_{1,2}^{\prime}$. The calculated integrals were analyzed in a within-subjects ANOVA with slant difference (the difference between the slant of the planar patch on the right and the slant of the planar patch on the left of the simulated surface; 7 levels) and axis position ( 2 levels) as the independent variables. The slant differences were 0 for the asymmetric surfaces (see the top panel of Figure 9) and $0.44,0.76,1.2,-0.44,-0.76$, and -1.2 for the asymmetric surfaces (see the bottom panel of Figure 9). Slant difference did not reach significance, $F(6$, $24)=1.793, n s$. There were a significant effect of the axis position, $F(1,4)=31.024, p<.01$, and a significant interaction between slant difference and axis position, $F(6$, 24 ) $=5.916, p<.01$ (see Figure 12). None of the other factors or interactions reached significance.

The top panels of Figure 13 show the judged depth differences as functions of the slant difference for each simulated axis of rotation. The plot on the left, for the two vertical probe dot distances $\left(Z_{0,1}^{\prime}-Z_{2,3}^{\prime}\right)$ indicates that when the axis of rotation was behind the surface, the difference between the judged depth separations was an increasing function of the slant difference. This result agrees with our predictions. Because we hypothesized that perceived depth separation is a decreasing function of slant, we expected that a greater difference between the slants of the two patches would result in a greater difference between the judged depths. However, when the axis of rotation was in front, the effect disappeared. This result can be explained by taking

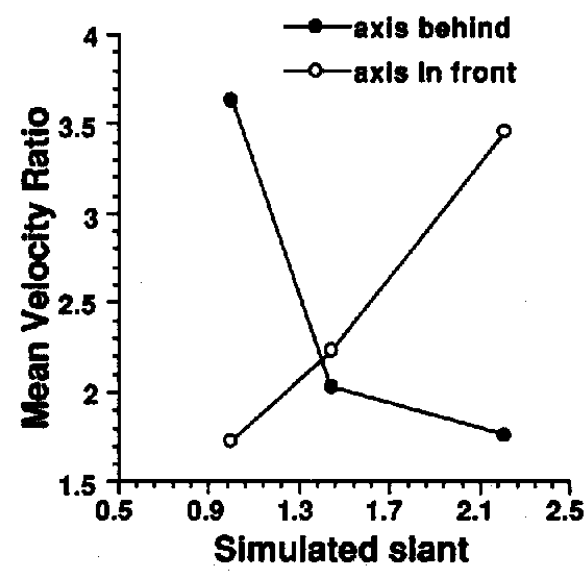

Figure 11. Mean ratio between the maximum and minimum projected velocities of the probe dots for the two simulated axes of rotation as a function of the slant of the lateral patch on which the pair of probe dots was located in Experiment 4.

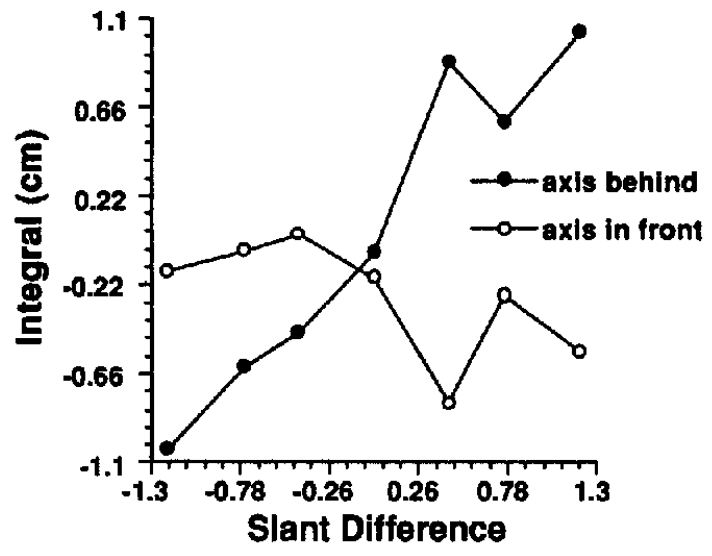

Figure 12. Mean integral of the depth judgments on a closed path for each simulated axis of rotation as a function of the signed difference between the slants of the lateral patches in Experiment 4.

into account the difference between the 2-D velocity ratios of the pair of probe dots $\mathbf{P}_{0}, \mathbf{P}_{1}$ and $\mathbf{P}_{2}, \mathbf{P}_{\mathbf{3}}$. The ratio between the 2-D velocities of a pair of probe dots was calculated by dividing the maximum velocity by the minimum velocity. The bottom left panel of Figure 13 shows the difference in velocity ratio as a function of the slant-difference for each axis position, for probe dots $\mathbf{P}_{0}, \mathbf{P}_{1}$ and $\mathbf{P}_{\mathbf{2}}, \mathbf{P}_{\mathbf{3}}$. When the axis of rotation was behind the surface, the velocity ratio of the probe dots on the less slanted planar patch was greater than the velocity ratio of the probe dots on the more slanted planar patch. As a consequence, the difference between the velocity ratios was an increasing function of the slant difference. The situation was reversed when the axis of rotation was in front. If perceived depth separation increases with velocity ratio, as well as with slant difference, the effects of these two variables would be in the same direction when the axis is behind the surface and opposite in direction when the axis is in front. This could result in an increased effect of slant difference in the former condition and a disappearance of that effect in the latter condition, accounting for the significant interaction shown in the upper left panel of Figure 13.

Let us consider the plot of the mean difference $Z_{0,3}^{\prime}-$ $Z_{1,2}^{\prime}$. If we hypothesize that only the pattern of slants of the surface on which two probe dots are located influences their perceived depth separation, we would expect that the differences between the perceived depth separations of the probe dots $\mathbf{P}_{\mathbf{0}}, \mathbf{P}_{\mathbf{3}}$ and $\mathbf{P}_{1}, \mathbf{P}_{\mathbf{2}}$ are null and do not depend on the position of the axis of rotation. However, the results plotted on the top right panel of Figure 13 indicate that this was not the case. When the axis of rotation was behind the surface, the mean difference between the judged depth separations was significantly greater. However, the effect of the position of the axis of rotation can also be explained by considering the plot of the velocity ratio difference (see the bottom right panel of Figure 13).

In conclusion, the results regarding the integral of the depth judgments along closed paths can be explained by considering the effects of two variables: the slant difference 

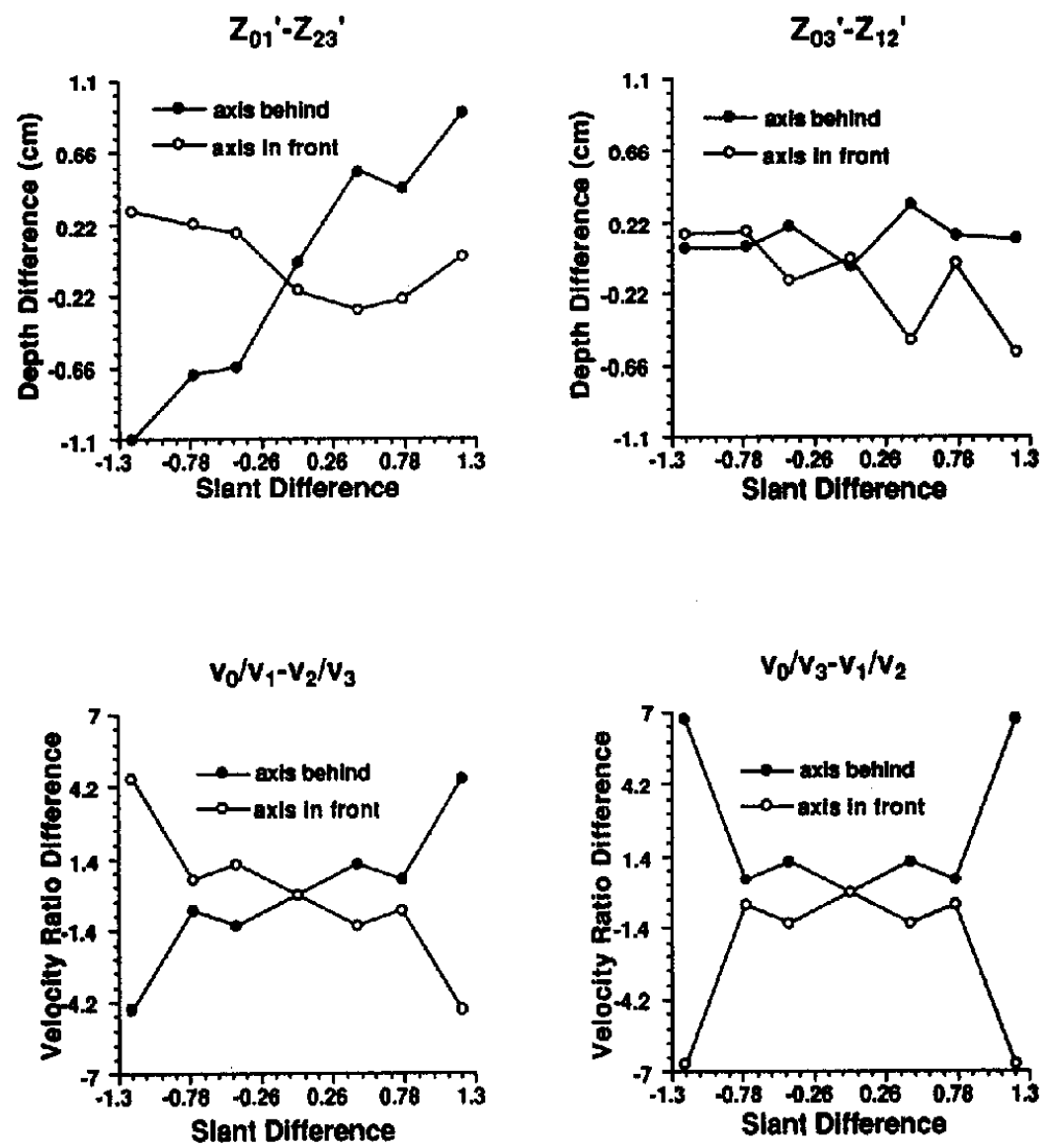

Figure 13. The top panels show the judged depth differences of opposite pairs of probe dots as a function of the signed difference between the slants of the lateral patches in Experiment 4 . The bottom panels show the difference in velocity ratio of opposite pairs of probe dots as a function of the signed difference between the slants of the lateral patches.

between the lateral planar patches and the velocity ratio of the probe dots. When the two variables cooperated, the integral of the depth judgments along closed paths increased as the asymmetry of the simulated surface increased. When the two variables were in conflict, the effect almost vanished. It is important to note that when the two variables cooperated, the depth judgments were inconsistent with a Euclidean model. When the two planar patches had slants of 0 and 2, for example, the mean integral was $1.0 \mathrm{~cm}$. This error should be considered large, because the mean of the depth judgments of the four pairs was $2.2 \mathrm{~cm}$.

The consistency of metric judgments has recently been studied by Koenderink et al. (1992) for surfaces of objects depicted in photographs. Observers in their study adjusted a gauge figure to fit the perceived local attitudes at a large number of positions sampled across a surface. Their results, unlike ours, suggest that local settings are internally consistent. We cannot compare our results with the results of Koenderink et al. directly, however, because their stimuli involved shape from shading and contours and not structure from motion. We cannot rule out the possibility that the perceptual system adopts different processes for global integration of local 3-D structure measurements when it is provided with different depth cues. The results of Norman et al. (1996), obtained with a procedure similar to the procedure used in the present experiment, support this possibility. Indeed, their results provide evidence that in a nearly full cue environment, the intrinsic structure of the perceptual space may be non-Euclidean.

\section{Experiment 5: Effect of Velocity Ratio}

The results of Experiment 4 indicate that the ratio between the 2-D velocities of two probe dots can influence their perceived depth separation. This variable, however, was not controlled in that experiment. Our purpose in Experiment 5 was to study directly the influence of the 2-D velocity ratio. Consider a planar surface rotating about the vertical axis and two pairs of probe dots on this surface that have the same simulated depth separations. If the two pairs project the same differences between the 2-D velocities, the ratios are, in general, different and depend on the distance of the pairs of dots from the axis of rotation: The greater the distance, the smaller the ratio. We should therefore expect 
that the perceived depth separations of the two pairs of probe dots will be different when the dots are at different distances from the axis of rotation, even when the same planar surface passes through the two points and the simulated slant (and def) is thus constant.

\section{Method}

Observers. The 5 observers who participated in Experiments 2 through 4 participated in this experiment.

Design. Two independent variables were examined: the slant of the planar surface $(1.0,1.44$, and 2.21) and the position of the two probe dots (four positions). The variables were run within observers.

Apparatus. The apparatus was the same as that in Experiment 1. Stimuli. The displays were similar to those in Experiment 4 except that planar surfaces were simulated and the axis of rotation was behind the surface in all conditions. The vertical gradient of the simulated planar surfaces was 1 in all the conditions. The slant of the planar surfaces was manipulated by changing the horizontal gradient; the slant could take the values of $1.0,1.44$, and 2.21 . To describe the possible positions of the probe dots on the image plane, we can refer to the right panel of Figure 8 . The only difference is that the vertical separation of the dots $\mathbf{P}_{\mathbf{0}}, \mathbf{P}_{\mathbf{1}}$ and $\mathbf{P}_{\mathbf{2}}, \mathbf{P}_{\mathbf{3}}$ was $14.4 \mathrm{~cm}$ in the present experiment. The simulated depth separation of the pairs of probe dots $P_{0}, P_{1}$ and $P_{2}, P_{3}$ was $14.0 \mathrm{~cm}$ in all the conditions. The simulated depth separation of the pairs of probe dots $\mathbf{P}_{0}, \mathbf{P}_{3}$ and $\mathbf{P}_{1}, \mathbf{P}_{\mathbf{2}}$ depended on the slant of the simulated planar surface on which the probe dots were positioned and could take values of $0,19.4$, and $36.7 \mathrm{~cm}$ for slants of $1,1.44$, and 2.21 , respectively (see Figure 14 ). The probe dots $\mathbf{P}_{0}$ and $\mathbf{P}_{1}$ were simulated at the same distances from the axis of rotation in all the conditions and projected the same 2-D velocities $\left(v_{0}=0.98 \%\right.$, $v_{1}=0.14 \%$, ratio $=7$ ). The ratio between the projected 2-D velocities of the dots $\mathbf{P}_{\mathbf{2}}$ and $\mathbf{P}_{\mathbf{3}}$ depended on the slant of the simulated planar surface and could take values of $7,1.64$, and 1.35 for slants of $1,1.44$, and 2.21 , respectively. The ratio between the projected 2-D velocities of the other two pairs of dots also depended on the slant of the simulated planar surface. For the $\mathbf{P}_{\mathbf{0}}, \mathbf{P}_{\mathbf{3}}$ pairs, the ratio could take on values of $1.0,9.34$, or 16.76 for slants of $1,1.44$, and 2.21 , respectively. For the $\mathbf{P}_{1}, \mathbf{P}_{\mathbf{2}}$ pairs the corresponding ratio values were $1.0,2.19$, or 3.25 .

Procedure. The procedure was the same as that in Experiment 4. The observers participated individually in two sessions of $\mathbf{4 8}$ trials presented in random order.

\section{Results and Discussion}

We conducted two separate ANOVAs on the judged depth separations for the pair of probe dots $\mathbf{P}_{0}, \mathbf{P}_{1}$ and $\mathbf{P}_{\mathbf{2}}, \mathbf{P}_{\mathbf{3}}$ and the pair $\mathbf{P}_{0}, \mathbf{P}_{3}$ and $\mathbf{P}_{1}, \mathbf{P}_{2}$. Both were 3 (slant) $\times 2$ (probe pair) within-subjects ANOVAs. For the probe pairs $\mathbf{P}_{\mathbf{0}}, \mathbf{P}_{\mathbf{1}}$ and $\mathbf{P}_{\mathbf{2}}, \mathbf{P}_{3}$, there was a significant effect of slant, $F(2,8)=9.401$, $p<.05$, and of probe pair, $F(1,4)=13.624, p<.05$. The interaction was also significant, $F(2,8)=15.494, p<.01$. For the probe pairs $\mathbf{P}_{\mathbf{0}}, \mathbf{P}_{\mathbf{3}}$ and $\mathbf{P}_{\mathbf{1}}, \mathbf{P}_{\mathbf{2}}$, there was a significant effect of slant, $F(2,8)=17.107, p<.01$, and of probe pair, $F(1,4)=9.686, p<.05$. Their interaction also reached significance, $F(2,8)=11.622, p<.01$. In an additional analysis we calculated the "reliability" of the judgments in each condition of Experiment 5 (see Appendix B).

Mean judged depth for each slant and for each pair of probe dots is plotted in Figure 15 as a function of slant. The left and right panels show the plots for the pair $\mathbf{P}_{\mathbf{0}}, \mathbf{P}_{\mathbf{1}}$ and $\mathbf{P}_{2}, \mathbf{P}_{3}$ and the pair $\mathbf{P}_{6}, \mathbf{P}_{3}$ and $\mathbf{P}_{1}, \mathbf{P}_{2}$, respectively. Consider first the judged depth separations for the pairs of probe dots $\mathbf{P}_{0}, \mathbf{P}_{1}$ and $\mathbf{P}_{2}, \mathbf{P}_{3}$ (see the left panel of Figure 15). The hypothesis that motivated the present experiment was confirmed: The ratio between the velocities of two probe dots influenced the judged depth separation. Indeed, when the slant of the planar surface was 1.44 or 2.21 , the mean judged
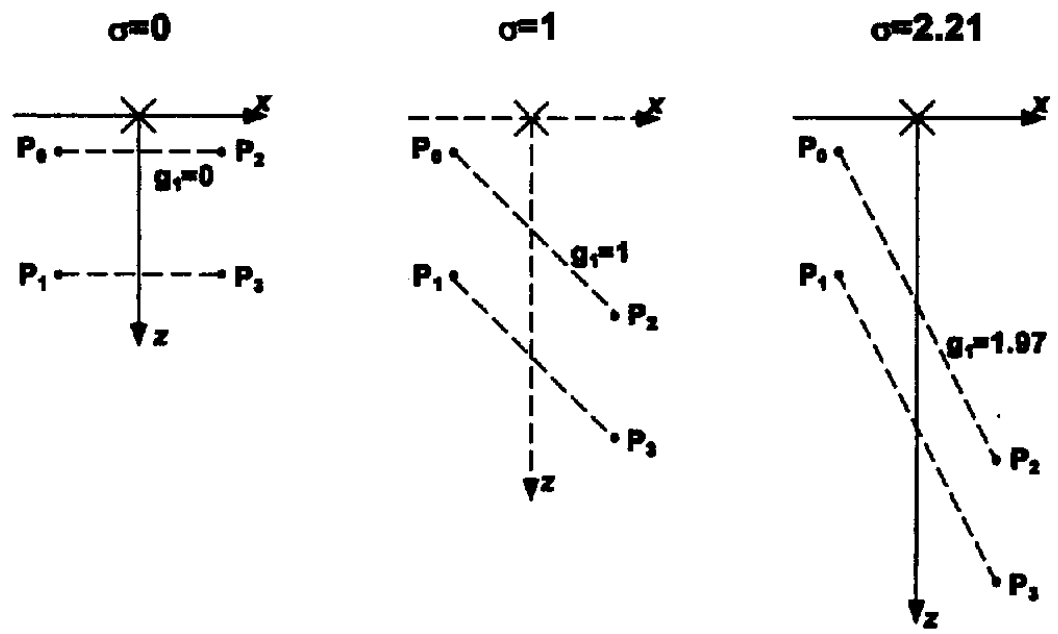

Figure 14. View from above of the simulated planar surfaces and probe dots in each condition of Experiment 5. The depth separations of the pairs of probe dots $\mathbf{P}_{\mathbf{0}}, \mathbf{P}_{\mathbf{1}}$ and $\mathbf{P}_{2}, \mathbf{P}_{3}$ are the same and do not change across conditions. The distances of $\mathbf{P}_{0}$ and $\mathbf{P}_{3}$ from the axis of rotation are the same and also do not vary across conditions. The distances of $\mathbf{P}_{\mathbf{2}}$ and $\mathbf{P}_{\mathbf{3}}$ from the axis of rotation increase with the slant $\sigma$ of the simulated planar surface. The slant $\sigma$ is varied by varying the horizontal component of the depth gradient $\left(g_{1}\right)$ while keeping the vertical component of the depth gradient $\left(g_{2}\right)$ equal to 1 . 

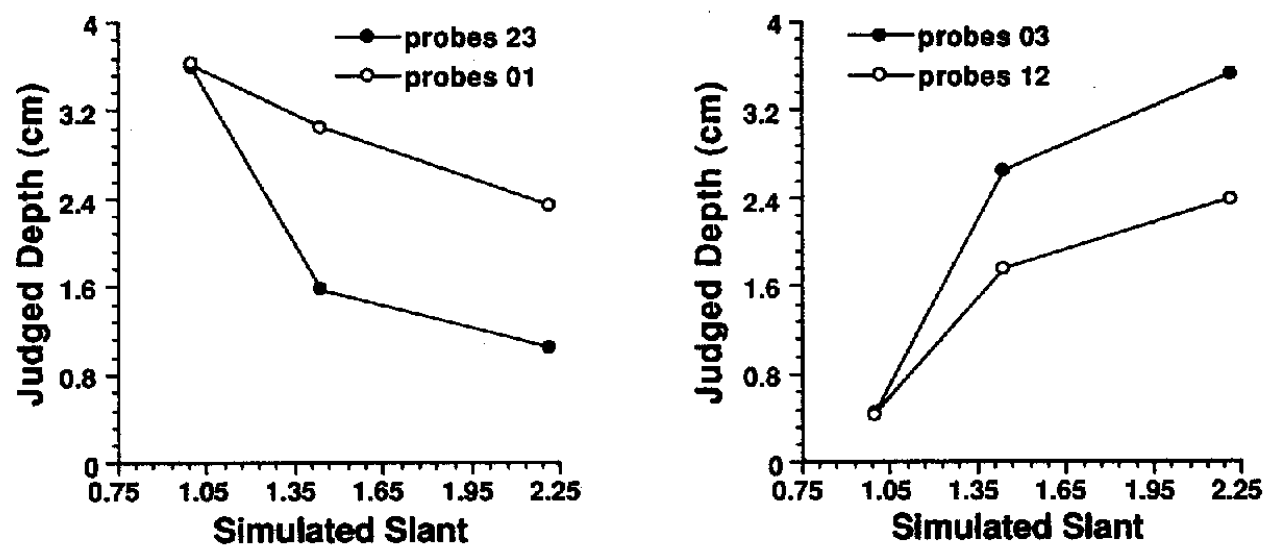

Figure 15. Mean judged depth for each pair of probe dots as a function of the simulated slant in Experiment 5.

depth separation of the pair $\mathbf{P}_{2}, \mathbf{P}_{\mathbf{3}}$ was half the mean judged depth separation of the pair $\mathbf{P}_{0}, \mathbf{P}_{1}$. Because the simulated depth separation and, therefore, the difference between the 2-D velocities was the same for both pairs of probe dots, the difference between the judged depth separations on the same simulated planar surface can only be attributed to the greater velocity ratio of the pair $\mathbf{P}_{0}, \mathbf{P}_{1}$.

We also replicated the results of Experiment 1, because the judged depth separation of the probe dots $\mathbf{P}_{0}, \mathbf{P}_{1}$ decreased as the simulated slant increased across surfaces. In this case, only the slant of the surface varied, because the 2-D velocities of the dots $\mathbf{P}_{0}$ and $\mathbf{P}_{1}$ were the same in all the conditions.

The judged depth separations for the pairs of probe dots $\mathbf{P}_{0}, \mathbf{P}_{3}$ and $\mathbf{P}_{1}, \mathbf{P}_{2}$ were also influenced by the ratio of the 2-D velocities (see the right panel of Figure 15). The mean judged depth separation of the pair of probe dots $\mathbf{P}_{\mathbf{1}}, \mathbf{P}_{\mathbf{2}}$ was $25 \%$ smaller than the mean judged depth separation of the pair of probe dots $\mathbf{P}_{\mathbf{0}}, \mathbf{P}_{\mathbf{3}}$ when the slant of the planar surface was 1.44 or 2.21. Because slant covaried with simulated depth separation, however, we cannot reach any conclusion about the effect of slant for these pairs of probe dots.

The results of the present experiment confirmed the hypothesis that the velocity ratio influences the perception of depth separation of two probe dots. It is likely that the difference in results when the axis of rotation was in front and when it was behind the simulated curved surfaces in Experiment 4 was due to the effects of this variable. (It is important to note that these results do not affect the conclusions for the first three experiments on the influence of slant on the perceived depth separation, because for each simulated depth separation in those experiments, the velocity ratio of the probe dots was kept constant.)

\section{Experiment 6: Depth Matching}

In Experiment 4 we found that the judged depth separations of two pairs of probe dots lying on the same simulated curved surface and having the same simulated depth separations could be different. These results indicate that, in general, perceived structure from motion is not a linear stretching along the line of sight of the simulated structure. In Experiment 4, however, two variables contributed to the results: the simulated slant of the planar surfaces on which the probe dots were positioned and their 2-D velocity ratio. In Experiment 5 we isolated the effect of the 2-D velocity ratio. Our purposes in Experiment 6 were to isolate the effect of the slant by keeping the velocity ratio constant and to test the same hypothesis that motivated Experiments 1,2, 3, and 4 with a different method. In Experiment 6, two pairs of probe dots were present in each display but were located on separate planar patches that rotated rigidly about a common vertical axis. The observer's task was to adjust the simulated relative depth between one pair of probe dots (the test pair) until the perceived depth separation was the same as the perceived depth separation of the other two probe dots (the comparison pair). If the slant of a planar surface that passes through two probe dots influences their perceived depth separation, as the results of Experiment 1 indicate, we should expect observers to be accurate when the slants of the comparison and test surfaces are equal and to make systematic errors when the simulated slants are different.

\section{Method}

Observers. The 5 observers who participated in Experiments 2 through 5 participated in this experiment.

Design. The horizontal component of the depth gradient was manipulated in four conditions: $(0.0,0.0),(0.0,2.0),(2.0,0.0)$ and $(2.0,2.0)$, where the two numbers in each pair are the horizontal components of depth gradient of the comparison and test patches, respectively. The conditions variable was varied within observers.

Apparatus. The apparatus was the same as that in Experiments 1 through 5 except that a separate response display was not used in the present experiment. Instead, the joystick was used to directly adjust the simulated depth separation of two probe dots in the stimulus display.

Stimuli. The displays were composed of light red dots on a black background. The four probe dots were light green dots. For each display, 200 dots were positioned randomly in two circular regions having diameters of $10.5 \mathrm{~cm}$ and having centers separated 
horizontally by $8.0 \mathrm{~cm}$. The right panel of Figure 16 shows the two circular regions; the left panel shows a schematic representation of the simulated structure. The motion of the dots simulated the orthographic projection of a 3-D rigid structure composed of two planar surfaces rotating in 3-D space about the same vertical axis through $\pm 6^{\circ}$. Unlike in Experiments 1 through 5, the boundaries did not extend beyond the viewing areas. The two simulated planar surfaces (the comparison and the test surface) projected to two circular regions at the midpoint of the rotation, and their projected contours deformed during the rotation. One entire cycle of rotation took $2 \mathrm{~s}$. The horizontal component of the depth gradient of the comparison surface could be 0.0 or 2.0 (see Figure 17). When the horizontal component was 0.0 , the vertical component was 0.5 . When the horizontal component was 2.0 , the vertical component was 1.0. The horizontal component of the depth gradient of the test surface also could be 0.0 or 2.0 . The vertical component was randomly selected between 0.0 and 2.0. At the midpoint of the rotation cycle, the two pairs of probe dots were centered in the two circular regions and the vertical separation of the probe dots in each pair was $8.7 \mathrm{~cm}$ (see Figure 16). The simulated depth separations of the comparison probe dots were $4.3 \mathrm{~cm}$ and $8.7 \mathrm{~cm}$ when the comparison surface had a horizontal depth gradient equal to 0.0 and 2.0 , respectively. The simulated depth separation for each pair of probe dots on the test surface was random and depended on the simulated vertical component of the depth gradient of the test surface (the range of depths was from 0 to $17.4 \mathrm{~cm}$ ). The velocities of the probe dots in each pair were equal in magnitude but opposite in sign (resulting in absolute velocity ratios of 1 ). Pressure on the joystick changed the vertical gradient of the test surface and, as a consequence, changed the simulated depth separation of the test probe dots and the slant of the surface (because the slant is the square root of the sum of squares of the vertical and horizontal gradients). Although this changed the velocities of the probe dots on the test surface, the velocities of the probe dots in each pair remained equal in magnitude, and the ratio of the absolute velocities of the probe dots in each pair remained equal to 1 .

Procedure. The observers were told that two planar surfaces rotating about a central vertical axis would be simulated. Their task was to adjust the relative depth between two test probe dots lying on one surface until their perceived depth separation was the same as the perceived depth separation of two comparison probe dots lying on the other surface. Observers were told that pushing the joystick forward (away from them) would increase the simulated depth of the test probe dots and that pulling the joystick backward (toward them) would decrease the simulated depth. Coarse changes in the simulated depth ( 10 times the change produced by moving the joystick's handle) could be made by pushing one of the joystick's buttons. The joystick's trigger button was pressed to initiate the next trial. The observers participated individually in two sessions of 80 trials presented in random order. For 2 observers, the comparison surface was on the left, and for 3 observers, it was on the right of the stimulus display.

\section{Results and Discussion}

A 2 (comparison surface) $\times 2$ (test surface horizontal gradient) within-subjects ANOVA was performed on the ratio between the adjusted depth separation of the test pair of probe dots and the simulated depth separation of the comparison pair of probe dots. There were significant effects of the comparison surface, $F(1,4)=13.309, p<.05$, and of the test surface horizontal gradient, $F(1,4)=10.104$, $p<.05$. Their interaction did not reach significance. In an additional analysis we calculated the "reliability" of the judgments in each condition of Experiment 6 (see Appendix B).

Table 1 shows the ratios between the adjusted depths of the test pair of probe dots and the simulated depths of the comparison pair of probe dots in the four experimental conditions, where $g_{1,1}$ and $g_{2,1}$ are the horizontal components of the depth gradients of the comparison and test surfaces and $s_{1}$ and $s_{2}$ are the slants of the comparison and test surfaces. Because the slant of the test surface was randomly selected at the beginning of each trial and changed during each trial as the observer adjusted the depth separation of the two probe dots, we report the slant that the surface would have when the adjusted depth separation was the same as the simulated depth separation of the comparison pair. $\Delta z$ is the simulated depth separation of the comparison probe dots.

Let us consider the conditions in which the horizontal components of the depth gradient were different for the

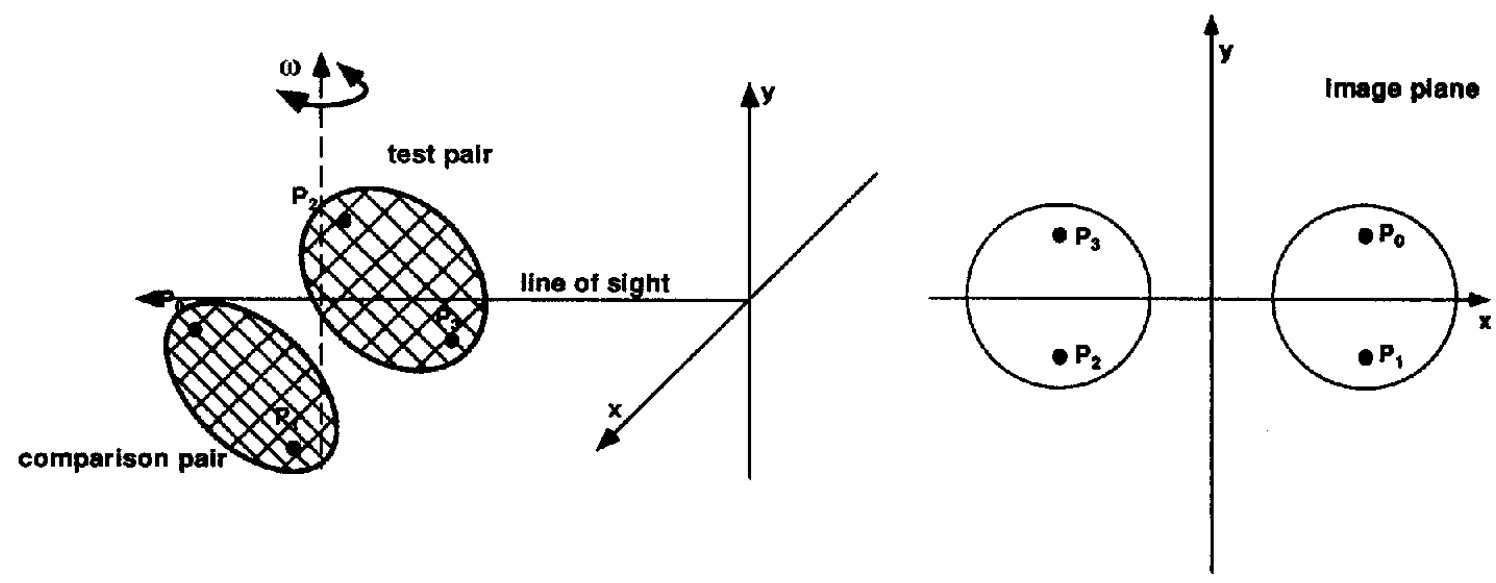

Figure 16. The simulated structure in Experiment 6 (left panel) and the projected circular regions of the two simulated planar surfaces (the comparison and the test surface) at the midpoint of the rotation cycle (right panel). 

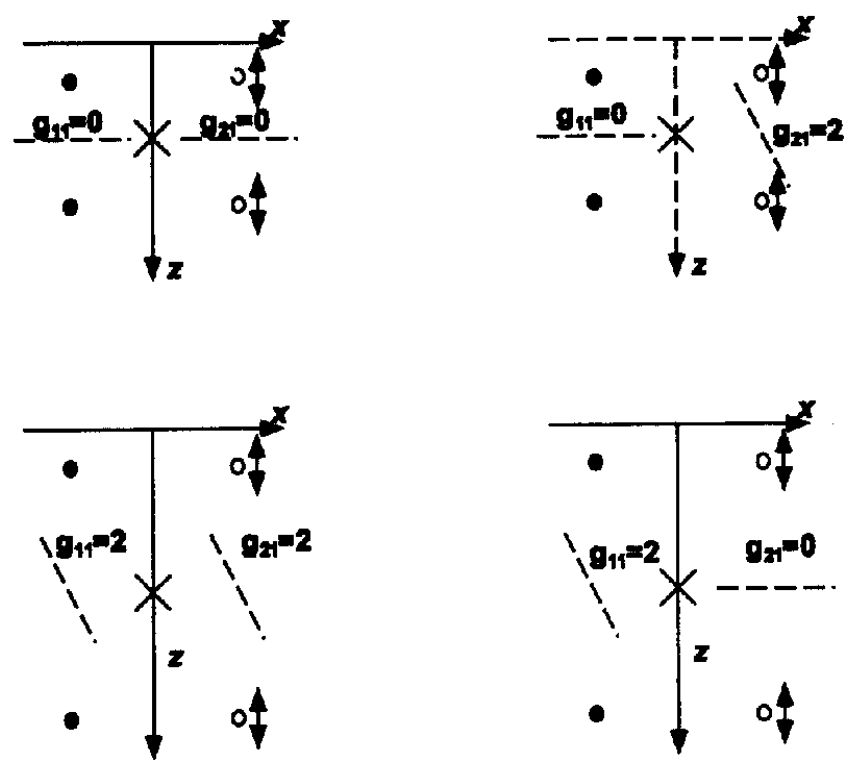

Figure 17. View from above of the simulated planar surfaces and probe dots in each condition of Experiment 6. The filled circles represent the comparison pair, whereas the open circles represent the test pair. The dashed lines represent the horizontal component of the depth gradient of the comparison $\left(g_{1,1}\right)$ and test $\left(g_{2,1}\right)$ surfaces.

comparison and test surfaces. When the horizontal gradient of the comparison surface was smaller than that of the test surface, the adjusted depth separation of the test probe dots was more then twice the simulated depth separation of the comparison pair of probe dots (ratio $=2.51$ ). This result agrees with the results of Experiment 1: The greater the simulated slant of the surface that passes through two probe dots, the smaller the perceived depth separation of the probe dots. Because the horizontal gradients of the test and comparison surfaces were different $(0.0$ and 2.0 ; see Table 1 ), the same simulated depths for the test and comparison pairs corresponded to different slants $(0.5$ and 2.06 ; see Table 1). From the results of Experiment 1 we would expect that when the two simulated depths were equal, the perceived depth of the test pair would be smaller than the perceived depth of the comparison pair, because the slant of the test pair was greater than the slant of the comparison pair. We can therefore infer that the observer adjusted a greater depth for the test pair in order to perceive the same depth for the two pairs of probe dots.

When the horizontal gradient of the comparison surface was greater than the horizontal gradient of the test surface, the adjusted depth of the test pair was smaller than the simulated depth of the comparison pair (ratio $=0.62$; see Table 1). In this case, the same simulated depths corresponded to slants of 2.23 and 1.0 for the comparison and test pairs, respectively (see Table 1). The same simulated depths would therefore correspond to a perceived depth of the test pair greater than the perceived depth of the comparison pair, because the slant of the test pair is smaller than the slant of the comparison pair. We can therefore conclude that in order to perceive the same depth separation for the two pairs of probe dots, the observers adjusted the depth separation of the test pair to be smaller than the simulated depth separation of the comparison pair. These results confirm the hypothesis that motivated the present experiment: The derived structure from orthographic projections of a moving rigid object is not a linear stretching of the simulated structure. When the slants of the surfaces that passed through the two pairs of probe dots were different, the observers adjusted the simulated depth separation of the test pair to be $251 \%$ greater or $38 \%$ smaller than the simulated depth separation of the comparison pair, in order to perceive the same depth separation. It is important to note that metric knowledge was not required to perform the task. Indeed, it would have been sufficient to adjust the test pair until the two imaginary lines that passed through the two pairs of probe dots were perceived as parallel. It is also important to point out that a rigidity assumption is not necessary to perform the task required of observers in the present experiment. In fact, algorithms for the affine derivation of structure from motion assume only that the projected structure undergoes a 3-D affine transformation (see Koenderink \& van Doorn, 1991).

When the horizontal gradients of the comparison and test surfaces were the same, however, we expected that the observers would correctly match the depth separations (i.e., the ratio would be 1.0 ). In these conditions, if the adjusted depth was the same as the comparison depth, the slants of the two surfaces would also be the same. The observers were almost correct in the condition in which the two horizontal gradients were 2.0 (ratio $=1.09$ ). This was not the case, however, when the two horizontal gradients were 0.5 . In this condition, the ratio of the adjusted depth to the comparison depth was 1.44. One possible explanation of the deviation of the obtained result from the expected result in the 0.5 condition is that the responses in this condition were biased by a range effect. The initial depth separation of the test probe dots was selected randomly on each trial within a range from 0 to $17.4 \mathrm{~cm}$. This range was used for all conditions. A correct match when the horizontal gradients were 0.5 would be $4.3 \mathrm{~cm}$. It is possible that a bias toward the center of the range could have led to the higher-thanexpected mean response $(6.2 \mathrm{~cm})$. Indeed, when the horizontal gradient was 2.0 and the correct adjusted depth was near the center of the range $(8.7 \mathrm{~cm})$, observers were nearly correct.

\section{Control Experiment}

Our purpose in conducting the control experiment was to investigate whether the biases in the results of Experiment 6

Table 1

Experiment 6 Stimulus Conditions and Results

\begin{tabular}{cccccc}
\hline$g_{1,1}$ & $g_{2,1}$ & $\Delta z$ & $s_{1}$ & $s_{2}$ & Ratio \\
\hline 0.0 & 0.0 & $4.3 \mathrm{~cm}$ & 0.50 & 0.50 & 1.44 \\
0.0 & 2.0 & $4.3 \mathrm{~cm}$ & 0.50 & 2.06 & 2.51 \\
2.0 & 0.0 & $8.7 \mathrm{~cm}$ & 2.23 & 1.00 & 0.62 \\
2.0 & 2.0 & $8.7 \mathrm{~cm}$ & 2.23 & 2.23 & 1.09 \\
\hline
\end{tabular}


could be attributed to the specific adjustment method used in that experiment. Observers responded to stimulus conditions selected on the basis of the results of Experiment 6 in a paired comparison design.

Three observers who had participated in Experiment 6 participated in the control experiment. Three independent variables were examined: horizontal gradients (same or different), depth separations (same or different), and surface position (left or right). The stimuli were a subset of the stimuli in Experiment 6 . The simulated gradients of the two surfaces and the depth separations of the two pairs of probe dots are shown in Table 2. $g_{1,1}$ and $g_{2,2}$ and $s_{1}$ and $s_{2}$ indicate the horizontal depth gradients and the slants, respectively, of the two surfaces. $\Delta z_{1}$ and $\Delta z_{2}$ are the simulated depth separations of the two pairs of probe dots.

As indicated in Table 2, one surface (the comparison surface) had the same slant and the same depth separation between the probe dots in all the conditions. In two experimental conditions, the simulated depth separations of the probe dots on the test and comparison surfaces were the same. In these conditions, the slants of the test and comparison surfaces could either be the same or different. When the simulated depth separations of probe dots on the test and comparison surfaces were different, the horizontal gradients could be the same or different. When the horizontal gradients of the comparison and test surfaces were 0.0 , we simulated a depth separation of the test probe dots that was $40 \%$ greater than the simulated depth separation of the comparison probe dots, because in Experiment 6 the adjusted mean test depth separation was $44 \%$ greater in the same condition. When the horizontal gradient of the test surface was 2.0 , we simulated a depth separation of the test probe dots that was $200 \%$ of the simulated depth separation of the comparison probe dots. The observers were asked to judge which pair of probe dots had the greater depth separation. If the pair on the left was perceived as having the greater depth separation, observers were to press the left button; otherwise they were to press the right button. Each observer participated individually in four sessions of 80 trials.

In each condition we calculated the percentage of responses in which the depth separation of the test probe dots was judged smaller than the depth separation of the comparison probe dots. These percentages are indicated in Table 2 . When the horizontal gradients were 0.0 and the simulated depth separations were the same, the observers were at chance in their responses. However, when the horizontal gradients were 0.0 and the simulated depth separations were different, observers indicated $67 \%$ of the time that the simulated depth separation was greater. Because the depth separation of the test pair was $40 \%$ greater than the depth separation of the comparison pair, it appears that observers' adjustment of the test pair to be $44 \%$ greater in Experiment 6 , when instructed to make the depth separations appear equal, was an artifact of the method used in that experiment. When the horizontal gradients of the two surfaces were different and the simulated depth separations of the two pairs of probe dots were the same, the observers judged the comparison pair depth separation to be greater than the test pair depth separation $94 \%$ of the time. This result confirms the results of Experiment 1: The greater the slant of a surface that passes through two probe dots, the smaller the perceived depth separation. When the horizontal gradients of the two surfaces and the simulated depth separations were different, the observers indicated $75 \%$ of the time that the comparison pair depth separation was greater even though the simulated depth separation of the comparison pair was half the simulated depth separation of the test pair.

\section{General Discussion}

The results of the six experiments reported here lead to two general conclusions about the perception of 3-D structure from motion: First, the mapping between a simulated 3-D structure and a perceived 3-D structure is not, in general, affine. Second, the perceptual representation of a 3-D structure derived from motion in a 2-D image is not, in general, Euclidean or affine. Evidence for the first conclusion is found in the results of the first three experiments, in which the judged depth separation of two probe dots varied with the slant of the surface passing through the probe dots or varied with the mean surface slant when more than one surface passed through the probe dots. If judged depth separation varies with surface slant, even when the simulated depth separation is held constant, judged depth cannot be modeled as a uniform stretching of simulated depth.

An alternative interpretation of the results of Experiments 1 through 3 is that a uniform scaling factor is applied to each display but that this factor changes from display to display. This possibility was disconfirmed in Experiment 6, in which surface patches with different slants, rotating together rigidly, were presented simultaneously. Observers adjusted the simulated depth separation for a pair of probe dots on one surface patch until it appeared to match the simulated depth separation of probe dots on another patch. We found that two pairs of probe dots having different simulated depth separations were judged as having the same depth separation, in accordance with the results of Experiments 1 through 3 . We

Table 2

Control Experiment Stimulus Conditions and Results

\begin{tabular}{ccccccc}
\hline$g_{1,1}$ & $g_{2,1}$ & $s_{1}$ & $s_{2}$ & $\Delta z_{1}$ & $\Delta z_{2}$ & $\% \Delta z_{2}^{\prime}<\Delta z_{1}^{\prime}$ \\
\hline 0.0 & 0.0 & 0.5 & 0.50 & $4.3 \mathrm{~cm}$ & $4.3 \mathrm{~cm}$ & 51 \\
0.0 & 0.0 & 0.5 & 0.70 & $4.3 \mathrm{~cm}$ & $6.1 \mathrm{~cm}$ & 33 \\
0.0 & 2.0 & 0.5 & 2.06 & $4.3 \mathrm{~cm}$ & $4.3 \mathrm{~cm}$ & 94 \\
0.0 & 2.0 & 0.5 & 2.23 & $4.3 \mathrm{~cm}$ & $8.7 \mathrm{~cm}$ & 75 \\
\hline
\end{tabular}


can conclude, therefore, that the scaling factor that relates perceived and simulated depth separation for a pair of points in a rotating rigid surface is a function of the local slant of the patch on which the pair lies.

The influence of the slant of a planar surface on the perceived depth separation of two dots that are located on the surface is predicted by a model that derives the slant from a first-order temporal property of the optic flow called def (Domini et al., 1995, 1997). Def is a function of angular velocity and simulated slant. In the present article we investigated the nature of the function relating perceived slant to simulated slant, for a constant value of angular velocity. We showed that if the perceived slant is not linearly related to the simulated slant, the perceived depth separation is also a function of the simulated slant (see Equation 7 and Appendix A for a derivation). In Experiments 1, 2, and 3 and Experiment 6, def was manipulated by changing the slant of the simulated surfaces with the angular velocity held constant (see Equation 1). The results are consistent with the hypothesis that the derived slant is a nonlinear function of def. Furthermore, they indicate that the ratio $f($ def $) /$ def decreases with increasing def and, therefore, that $f(d e f)$ is a sublinear function of def.

Our results relating judged depth separation to def are consistent with results from a recent study showing a relationship between judged shape of a dihedral angle and the velocity gradients in orthogonal directions (Liter \& Braunstein, 1998). The simulated objects in that study were dihedral angles consisting of two planes slanted about a horizontal axis and meeting at a horizontal edge. Different combinations of motion and projection were studied, including perspective projections of translations with the dihedral edge either frontal parallel or rotated about the vertical axis and orthographic projections of rotations about a vertical axis. We computed def for the slanted planes comprising the dihedral angles in each condition in that study. Mean values of $d e f$ were used for the rotated translations and rotations, because def varies over views for these conditions. The value of def was greatest for the frontal translations and about the same for the other two conditions, though the mean def for rotations was greater than the mean def for rotated translations. In Appendix $A$ of the present article we show mathematically that if the perceived slant is a sublinear function of def, then the judged dihedral angle is an increasing function of def. This theoretical finding is consistent with the results of Liter and Braunstein (1998), because observers judged the frontal translations as representing steeper angles (more depth relative to height) than the rotated translocations or rotations. Domini et al. (1997) also found that the perceived magnitude of angular displacement (i.e., the perceived amount of rotation) was an increasing function of def. These results are consistent with Liter and Braunstein's finding that the rotations and the rotated translations were judged as undergoing greater angular displacements than the frontal translations, although in fact the rotated translations, like the frontal parallel translations, displayed a null angular displacement.

Further evidence for our first general conclusion, that the mapping is not affine, is found in the results of Experiments
4 and 5. The results of Experiment 4 suggest that a second factor, the ratio of the velocities of the probe dots, affects judged depth separation when simulated depth separation is held constant. The effect of the velocity ratio was clearly demonstrated in Experiment 5 when def was held constant for pairs of probe dots differing in velocity ratio. The effect of velocity ratio on judged depth separation is inconsistent with an orthographic analysis that maps simulated depth onto judged depth through uniform scaling. The effect of velocity ratio is consistent with a perspective analysis of the image: In a rigid rotation, with the vertical separation in the image and the velocity difference in the image fixed for two pairs of probe dots, a geometric analysis based on parallel projection would yield the same depth separation for each pair, but if the velocity ratios differed between pairs an analysis based on perspective projection would indicate a greater depth separation between the points with the greater velocity ratio. The conclusion that a perspective analysis may be applied in the perception of orthographic projections was reached by Braunstein et al. (1993) and Liter and Braunstein (1998) with different stimuli and methods.

Although the second general conclusion, that the representation is not Euclidean or affine, might be derived theoretically from the first conclusion, Experiment 4 provides direct evidence for the second conclusion. If the perceived 3-D structure could be represented in Euclidean space, or even in affine geometry, the algebraic sum of metric distances along a closed path in depth would have to vanish. In the axis-behind condition of Experiment 4 we found that judgments on closed paths on a curved surface were not internally consistent, because the integral of the judgments did not vanish when the structure was asymmetric, that is, when the two planar patches on the lateral regions of the curved surface had different slants. This was because the perceived depth separations of pairs of probe dots that were located on differently slanted regions were different. It should be noted that the derivation of the correct relative depth separations of the two pairs of probe dots does not require metric knowledge, because the directions along which the measurements had to be made are parallel (Koenderink \& van Doorn, 1991). In Experiment 4 not only were the required judgments in the same direction (along the line of sight) but also imaginary lines connecting the probe dots on the two lateral planar patches were parallel.

Although the present results are inconsistent with affine mapping or representation in structure from motion, they are consistent with the view that a first-order temporal analysis of the optic flow is used by human observers in SFM tasks (Todd et al., 1988; Todd \& Bressan, 1990; Todd \& Norman, 1991). In fact, both the nonlinear model and the velocity ratio computation require only the information available in two views. Although two views are not sufficient geometrically for a unique solution in structure from motion, they appear to be sufficient for a perception of depth separation that is directly predictable from the image properties but neither veridical nor internally consistent with respect to Euclidean geometry. Moreover, the results of the experiments described in this article indicate that the first-order temporal properties of the optic flow mainly influence 
human performance even if the number of views are sufficient for a mathematically correct analysis of the moving images. If the mapping of physical 3-D space to perceived 3-D space is not Euclidean and is not affine, what is the mapping? It is not clear that postulating a particular higher order geometry or set of geometries for either the mapping or the representation is necessary for an understanding of the recovery of 3-D structure from motion. (See J. F. Norman \& Todd, 1992, for a discussion of Klein's, 1893, hierarchy of geometries.) This does not imply, however, that perceived structure is not predictable from image motion. On the contrary, the present results, together with the results of Domini et al. (1997), Liter and Braunstein (1998), and Liter et al. (1993), suggest that specific heuristic processes ${ }^{5}$ relating image information to perceived depth and rigidity are used by the perceptual system to derive 3-D motion and shape from moving 2-D images. As we continue to develop an understanding of these processes, we will also increase our understanding of how perceived 3-D structure is related to image motion.

${ }^{5}$ See Braunstein (1994) for a discussion of heuristic processes in perception.

\section{References}

Bennett, B. M., Hoffman, D. D., Nicola, J. E., \& Prakash, C. (1989). Structure from 2 orthographic views of rigid motion. Journal of the Optical Society of America A, 6, 1052-1069.

Braunstein, M. L. (1994). Decoding principles, heuristics and inference in visual perception. In G. Jansson, S. S. Bergstrom, \& W. Epstein (Eds.), Perceiving events and objects (pp. 436-446). Hillsdale, NJ: Erlbaum.

Braunstein, M. L., Hoffman, D. D., Shapiro, L. R., Andersen, G. J., \& Bennett, B. M. (1987). Minimum points and views for the recovery of three-dimensional structure. Journal of Experimental Psychology: Human Perception and Performance, 13, 335343.

Braunstein, M. L., Liter, J. C., \& Tittle, J. S. (1993). Recovering 3-D shape from perspective translations and orthographic rotations. Journal of Experimental Psychology: Human Perception and Performance, 19, 598-614.

Caudek, C., \& Proffitt, D. R. (1993). Depth perception in motion parallax and stereokinesis. Joumal of Experimental Psychology: Human Perception and Performance, 19, 32-47.

Domini, F., Caudek, C., \& Gerbino, W. (1995). Perception of surface attitude in SFM displays. Investigative Ophthalmology and Visual Science, 36, \$360.

Domini, F., Caudek, C., \& Proffitt, D. R. (1997). Misperceptions of angular velocities influence the perception of rigidity in the kinetic depth effect. Joumal of Experimental Psychology: $\mathrm{Hu}$ man Perception and Performance, 23, 1111-1129.

Hildreth, C. H., Grzywacz, N. M., Adelson, E. H., \& Inada, V. K. (1990). The perceptual buildup of three-dimensional structure from motion. Penception \& Psychophysics, 48, 19-36.

Hoffman, D. (1982). Inferring local surface orientation from motion fields. Journal of the Optical Society of America, 72, 888-892.

Husain, M., Treue, S., \& Andersen, R. A. (1989). Surface interpolation in three-dimensional structure-from-motion perception. $\mathrm{Neu}$ ral Computation, 1, 324-333.

Indow, T. (1991). A critical review of Luneburg's model with regard to global structure of visual space. Psychological Review, $98,430-453$.

Klein, F. (1893). Vergleichende Betrachtungen ueber neuere geometrische Forschungen ("Erlanger Programm") [Comparative observations on recent research in geometry ("Erlanger program")]. Mathematische Annalen, 43, 63-100.

Koenderink, J. J. (1986). Optic flow. Vision Reseanch, 26, 161-179.

Koenderink, J. J., \& van Doorn, A. J. (1976). Local structure of movement parallax of the plane. Journal of the Optical Society of America, 66, 717-723.

Koenderink, J., \& van Doom, A. (1986). Depth and shape from differential perspective in the presence of bending deformations. Journal of the Optical Society of America A, 3, 242-249.

Koenderink, J. J., \& van Doom, A. J. (1991). Affine structure from motion. Journal of the Optical Society of America A, 8, 377-385.

Koenderink, J. J., van Doom, A. J., \& Kappers, A. M. L. (1992). Surface perception in pictures. Penception \& Psychophysics, 52, 487-496.

Liter, J. C., \& Braunstein, M. L. (1998). The relationship of vertical and horizontal velocity gradients in the perception of shape, rotation, and rigidity. Journal of Experimental Psychology: Human Perception and Performance, 24, 1257-1272.

Liter, J. C., Braunstein, M. L., \& Hoffman, D. D. (1993). Inferring structure from motion in two-view and multi-view displays. Perception, 22, 1441-1465.

Marr, D. (1982). Vision. San Francisco: Freeman.

Norman, F. N., Todd, J. T., Perotti, V. J., \& Tittle, J. S. (1996). The visual perception of three-dimensional length. Joumal of Experimental Psychology: Human Perception and Performance, 22, 173-186.

Norman, J. F., \& Todd, J. T. (1992). The visual perception of 3-D dimensional form. In G. A. Carpenter \& S. Grossberg (Eds.), Neural networks for vision and image processing (pp. 93-110). Cambridge, MA: MIT Press.

Tittle, J. S., Todd, J. T., Perotti, V. J., \& Norman, F. N. (1995). Systematic distortion of perceived three-dimensional structure from motion and binocular stereopsis. Journal of Experimental Psychology: Human Perception and Performance, 21, 663-678.

Todd, J. T., Akerstrom, R. A., Reichel, F. D., \& Hayes, W. (1988). Apparent rotation in three-dimensional space: Effects of temporal, spatial, and structural factors. Perception \& Psychophysics, $43,179-188$.

Todd, J. T., \& Bressan, P. (1990). The perception of 3-dimensional affine structure from minimal apparent motion sequences. Perception \& Psychophysics, 48, 419-430.

Todd, J. T., \& Norman, J. F. (1991). The visual perception of smoothly curved surfaces from minimal apparent motion sequences. Perception \& Psychophysics, 50, 509-523.

Todd, J. T., Tittle, J. S., \& Norman, J. F. (1995). Distortions of three-dimensional space in the perceptual analysis of motion and stereo. Perception, 24, 75-86.

Todorovic, D. (1993). Analysis of two- and three-dimensional rigid and nonrigid motions in the stereokinetic effect. Journal of the Optical Society of America A, 10, 804-826.

Ullman, S. (1979). The interpretation of visual motion. Cambridge, MA: MIT Press.

Verri, A., Girosi, F., \& Torre, V. (1990). Differential techniques for optical flow. Journal of the Optical Society of America, 7A, 912-922.

Wallach, H., \& O'Connell, D. N. (1953). The kinetic depth effect. Journal of Experimental Psychology, 45, 205-217.

Werkhoven, P., \& van Veen, H. A. H. C. (1995). Extraction of relief from visual motion. Perception \& Psychophysics, 57, 645-656. 


\section{Appendix A}

The Model

Consider a Cartesian coordinate system $(x, y, z)$ centered at the observer. A planar surface $\Pi$ can be described by

$$
f(x, y)=g_{1} x+g_{2} y+d,
$$

where $g_{1}$ and $g_{2}$ are the two components of the depth gradient of the plane $\Pi$ in the $x$ and $y$ directions, that is, the $x$ and $y$ components of a vector $\mathbf{N}$ orthogonal to the planar surface having the third component (the $z$ component) equal to 1 , and intersecting the $z$-axis at $d$. The tilt $(\tau)$ of the plane $I$ is defined as

$$
\tau=\frac{g_{2}}{g_{1}}
$$

and its slant $(\sigma)$ as

$$
\sigma=\sqrt{g_{1}^{2}+g_{2}^{2}}
$$

To better grasp the geometrical meaning of Equations A2 and A3, consider a generic plane $\Pi$ which intersects the $z$ axis at $d$. If we project $N$ on the $x-y$ plane, then we obtain a two-dimensional vector $\left(\mathrm{N}^{\prime}\right)$ whose components are $g_{1}$ and $g_{2}$. The tangent of the angle $\alpha$ (i.e., the angle between $N^{\prime}$ and the $x$-axis) is the tilt of the plane II. Thus, the tilt is the inclination of the projection of the normal vector $(\mathbf{N})$ on the $x-y$ plane. The modulus of $\mathbf{N}$ is the slant $\sigma$ of $\Pi$, that is, the inclination of $\Pi$ with respect to the $z$-axis. If $\Pi$ is parallel to the $x-y$ plane, then the vector $\mathbf{N}$ projects as a point. In this case the slant $\sigma$ of $\Pi$ is zero. When $\Pi$ is perpendicular to the $x-y$ plane, $\sigma$ takes on its maximum value (infinity). The angle between the normal vector $\mathbf{N}$ and the $z$-axis is the arc tangent of $\sigma$.

Let us assume that the plane $\Pi$ is undergoing a generic 3-D motion. The rotation components about the $x, y$, and $z$ axes of the global rotation vector $\Omega$ are $\dot{\phi}, \dot{\theta}$, and $\dot{p} ; \dot{\phi}$ and $\theta$ define the rotation component of $\boldsymbol{\Omega}$ in the $x-y$ plane. The modulus of this component, $\omega$, is given by

$$
\omega=\sqrt{\dot{\theta}^{2}+\dot{\phi}^{2}}
$$

The tilt of the axis of rotation of $\Pi, \tau_{\omega}$ (i.e., the inclination of the projection of the axis of rotation on the $x-y$ plane), is

$$
\tau_{\omega}=\frac{\dot{\theta}}{\dot{\phi}}
$$

If we let $t_{x}, t_{y}$, and $t_{z}$ represent the $x, y$, and $z$ components of the translation velocity of $\Pi$, then we can write the following matrix equation assigning a velocity vector $\mathrm{V}$ to every point $[x, y, f(x, y)]$ of $\Pi$ :

$$
\mathbf{V}=\left[\begin{array}{ccc}
0 & -\dot{\rho} & -\dot{\theta} \\
\dot{\boldsymbol{\rho}} & 0 & -\dot{\phi} \\
\dot{\theta} & \dot{\phi} & 0
\end{array}\right]\left[\begin{array}{c}
x \\
y \\
f(x, y)
\end{array}\right]+\left[\begin{array}{c}
t_{x} \\
t_{y} \\
t_{z}
\end{array}\right]
$$

From Equation A6 it follows that the $x$ and $y$ velocity components of the points of $\Pi$ are

$$
\begin{aligned}
& \mathbf{V}_{x}=t_{x}-y \dot{\mathbf{\rho}}-\left(g_{1} x+g_{2} y+d\right) \dot{\boldsymbol{\theta}} \\
& \mathbf{V}_{y}=t_{\mathrm{y}}+x \dot{\boldsymbol{\rho}}-\left(g_{1} x+g_{2} y+d\right) \dot{\phi}
\end{aligned}
$$

Equations A7 specify a linear vector field in the image plane. Hoffmann (1982) showed that the first-order properties of this velocity field are sufficient to determine (up to a reflection) the tilt of the axis of rotation of $\Pi, \tau_{\omega}$, the rotatory component of the angular velocity $\boldsymbol{\Omega}$ about the $z$ axis $\dot{\boldsymbol{p}}$, and the tilt $\tau$ of $I I$.

The partial derivatives of the velocity field with respect to the $x$ and $y$ axis are

$$
\mathbf{M}=\left[\begin{array}{ll}
\frac{\delta \mathbf{V}_{x}}{\delta x} & \frac{\delta \mathbf{V}_{x}}{\delta y} \\
\frac{\delta \mathbf{V}_{y}}{\delta x} & \frac{\delta \mathbf{V}_{y}}{\delta y}
\end{array}\right]
$$

In the case of the velocity field produced by the orthographic projection of the generic 3-D motion of the plane $\Pi$, the matrix $\mathbf{M}$ becomes

$$
\mathbf{M}=\left[\begin{array}{cc}
-g_{1} \dot{\theta} & -\dot{\boldsymbol{\rho}}-g_{2} \dot{\theta} \\
\dot{\boldsymbol{\rho}}-g_{1} \dot{\boldsymbol{\phi}} & -g_{2} \dot{\boldsymbol{\phi}}
\end{array}\right] \text {. }
$$

This matrix can be decomposed into a sum of four orthogonal matrices $\left(\mathbf{M}_{k}\right)$ multiplied by four coefficients $\left(C_{k}\right)$, the so-called differential invariants (Verri, Girosi, \& Torre, 1990):

$$
\mathbf{M}=\frac{1}{2} \sum_{k=1}^{4} C_{k} \mathbf{M}_{k}
$$

The coefficients $C_{k}$ characterize different types of 2-D elementary motions. The first one, div, identifies an isotropic expansion. The second one, curl, defines a local vorticity of the motion field. The last two coefficients, $\operatorname{def}_{1}$ and $d e f_{2}$, are the shear components along two orthogonal directions:

$$
\begin{array}{ll}
\text { div }=C_{1}=\frac{\delta \mathbf{V}_{x}}{\delta x}+\frac{\delta \mathbf{V}_{y}}{\delta y} & \mathbf{M}_{1}=\left[\begin{array}{ll}
1 & 0 \\
0 & 1
\end{array}\right] \\
\text { curl }=C_{2}=\frac{\delta \mathbf{V}_{y}}{\delta x}-\frac{\delta \mathbf{V}_{x}}{\delta y} & \mathbf{M}_{2}=\left[\begin{array}{ll}
0 & -1 \\
1 & 0
\end{array}\right] \\
\text { def } 1=C_{3}=\frac{\delta \mathbf{V}_{x}}{\delta x}-\frac{\delta \mathbf{V}_{y}}{\delta y} & \mathbf{M}_{3}=\left[\begin{array}{ll}
1 & 0 \\
0 & -1
\end{array}\right] \\
\text { def }_{2}=C_{4}=\frac{\delta \mathbf{V}_{x}}{\delta y}+\frac{\delta \mathbf{V}_{y}}{\delta x} & \mathbf{M}_{4}=\left[\begin{array}{ll}
0 & 1 \\
1 & 0
\end{array}\right]
\end{array}
$$


By substituting the entries of $\mathbf{M}$ (see Equation $A 9$ ) in Equation All, we obtain

$$
\begin{aligned}
& d i v=-g_{1} \dot{\theta}-g_{2} \dot{\phi} \\
& \text { curl }=2 \dot{\boldsymbol{\rho}}-g_{1} \dot{\phi}+g_{2} \dot{\theta} \\
& d e f_{1}=-g_{1} \dot{\theta}+g_{2} \dot{\phi} \\
& d e f_{2}=-g_{1} \dot{\phi}-g_{2} \dot{\theta},
\end{aligned}
$$

and by summing the squares of the last two equations, we obtain

$$
d e f_{1}^{2}+d e f_{2}^{2}=\left(g_{1}^{2}+g_{2}^{2}\right)\left(\dot{\phi}^{2}+\dot{\theta}^{2}\right) .
$$

Recalling Equations $\mathrm{A} 3$ and $\mathrm{A} 4$, we can rewrite Equation $\mathrm{A} 13$ as

$$
\sqrt{\operatorname{def}_{1}^{2}+\operatorname{def}_{2}^{2}}=\sigma \omega
$$

The square root of the sum of the squares of the shear components is called deformation (def).

One can also calculate def by considering three points and their 2-D velocities (see Figure 8). Let us rewrite Equation A7 in the following manner:

$$
\begin{aligned}
& \mathbf{V}_{x}=-\left(g_{1} \dot{\theta}\right) x-\left(g_{2} \dot{\theta}+\dot{\rho}\right) y+t_{x}-d \dot{\theta} \\
& \mathbf{V}_{y}=-\left(g_{1} \dot{\phi}-\dot{\rho}\right) x-\left(g_{2} \dot{\phi}\right) y+t_{y}-d \dot{\phi} .
\end{aligned}
$$

If the velocity components $\mathrm{V}_{x}$ and $\mathrm{V}_{y}$ and the coordinates $(x, y)$ of a point $P$ are known, then Equations $A 15$ can be considered as a system of two linear equations with six unknowns (i.e., the four coefficients of $x$ and $y$ and the two constants). To solve for the six unknowns, three distinct points are needed. The solution of the system of six equations gives us the four entries of the matrix described by Equations A8 and A9. Therefore, we can calculate the $\operatorname{def}_{1}$ and $\operatorname{def}_{2}$ components with Equation A11. Taking the square root of the sum of the squares of the two shear components, and rearranging the result, we can express def in the following manner:

$$
\begin{gathered}
d e f=\frac{1}{|\sin \alpha|} \\
\sqrt{\frac{\left(\frac{\left|\mathbf{V}_{1}-\mathbf{V}_{0}\right|}{\rho_{1}}\right)^{2}+\left(\frac{\mathbf{V}_{2}-\mathbf{V}_{0} \mid}{\rho_{2}}\right)^{2}}{-2 \frac{\left|\mathbf{V}_{1}-\mathbf{V}_{0}\right|}{\rho_{1}} \frac{\left|\mathbf{V}_{2}-\mathbf{V}_{0}\right|}{\rho_{2}} \cos \left(\alpha-\alpha_{\Delta}\right)}}
\end{gathered}
$$

where $V_{0}, V_{1}$, and $V_{2}$ are the velocity vectors of the projected points, $\rho_{1}$ and $p_{2}$ are the distances of the points $P_{1}$ and $P_{2}$ from $P_{0}, \alpha$ is the angle between the line segments $\mathbf{P}_{0} \mathbf{P}_{1}$ and $\mathbf{P}_{0} \mathbf{P}_{2}$, and $\boldsymbol{\alpha}_{\Delta}$ is the difference between the angles of the velocity vectors.

There is empirical evidence that the perceived slant $\left(\sigma^{\prime}\right)$ of a rotating planar surface is a monotonically increasing function of def and that the tilt $\left(\tau^{\prime}\right)$ is correctly derived (Domini, Caudek, \& Gerbino, 1995). We can therefore assume that

$$
\sigma^{\prime}=f(d e f)
$$

and

$$
\tau^{\prime}=\tau
$$

Let us consider the implications that these hypotheses have on the derivation of the depth separation of two dots located on a planar surface.

Without loss of generality, one point $P_{0}$ can be chosen as lying at the origin of a Cartesian system. Another point $\mathbf{P}_{\mathbf{1}}$ has generic coordinates $(x, y, z)$.

The equation that determines the infinite family of planar surfaces passing through the points $\mathbf{P}_{\mathbf{0}}$ and $\mathbf{P}_{\mathbf{1}}$ is

$$
z=g_{1} x+g_{2} y .
$$

If we substitute Equation $\mathrm{A} 2$ in Equation $\mathrm{A} 3$ we can write

$$
\sigma=\left|g_{1}\right| \sqrt{1+\tau^{2}},
$$

and by combining Equations $\mathrm{A} 2, \mathrm{~A} 19$, and $\mathrm{A} 20$ we obtain an expression for $z$ (the separation in depth between the points $P_{0}$ and $\left.\mathbf{P}_{1}\right)$ :

$$
z=\frac{\mathbf{\sigma}}{\sqrt{1+\tau^{2}}}(x+\tau y) .
$$

Because we hypothesized that the derived slant is a function of def (Equation 2) we can rewrite Equation A21 as

$$
z^{\prime}=\frac{f(d e f)}{\sqrt{1+\tau^{2}}}(x+\tau y) .
$$

The ratio between Equations $\mathrm{A} 22$ and $\mathrm{A} 21$ gives a simple relation between the simulated depth and the derived depth:

$$
z^{\prime}=z \frac{f(d e f)}{\sigma} .
$$

Taking into account Equation A14, we can rewrite this as

$$
z^{\prime}=z \omega \frac{f(\text { def })}{\operatorname{def}} .
$$

This equation shows the relationship between derived and simulated depth. It is possible, however, to relate the derived depth to the image characteristics directly. If we consider a rotation about an axis contained in the image plane, the depth separation $(z)$ between two points is related to the projected 2-D velocities $\left(V_{1}, V_{2}\right)$ of the points as follows (see Equation A15 for a derivation): $z \omega=$ $\left(V_{2}-V_{1}\right)$. Therefore, Equation A24 becomes

$$
z^{\prime}=\frac{f(\text { def })}{\operatorname{def}}\left(\mathbf{v}_{2}-\mathbf{v}_{1}\right)
$$

Relationship to the results of Liter and Braunstein (1998): The purpose of the following derivation is to show that the derived $\beta$ of a dihedral angle increases as the deformation projected by the two planar surfaces of the dihedral angle increases. 
The normal to the planar surface described by Equation $A 1$ is

$$
\mathbf{N} \equiv \frac{1}{\Delta}\left(g_{1}, g_{2},-1\right)
$$

where

$$
\Delta=\sqrt{g_{1}^{2}+g_{2}^{2}+1}
$$

The normal to the plane $x z$ (the horizontal plane) is

$$
\mathbf{N}_{x z} \equiv(0,1,0)
$$

The cosine of the angle between the horizontal plane and the planar surface is the inner product $\mathbf{N}_{x z} \cdot \mathbf{N}$ of the two normals:

$$
\cos \beta=\frac{g_{2}}{\sqrt{g_{1}^{2}+g_{2}^{2}+1}}
$$

Combining Equation A28 with Equation A3, we obtain

$$
\cos \beta=\frac{g_{2}}{\sqrt{\sigma^{2}+1}} .
$$

One can express $g_{2}$ in terms of tilt and slant by combining Equations $A 2$ and $A 3$ :

$$
g_{2}=\frac{\sigma}{\sqrt{\frac{1}{\tau^{2}}+1}}
$$

Let us consider the optic flow produced by a surface rotating about the vertical axis. Because the components of angular velocity parallel to the $x$ and $z$ axes are null, the optic flow is horizontal:

$$
\mathbf{V}_{x}=\left(-g_{1} \dot{\theta}\right) x+\left(-g_{2} \dot{\theta}\right) y+\left(d \dot{\theta}+t_{x}\right)
$$

The horizontal gradient $\left(\phi_{h}\right)$ of the resulting flow field is $-g_{1} \dot{\theta}$, and the vertical gradient $\left(\phi_{v}\right)$ is $-g_{2} \dot{\theta}$. In this simple case the tilt $\tau$ of the surface can be derived by taking the ratio between the vertical and the horizontal gradients:

$$
\tau=\frac{\phi_{\mathrm{v}}}{\phi_{\mathrm{h}}}=\frac{g_{2}}{g_{1}} .
$$

The deformation is simply the square root of the sum of the squares of the horizontal and vertical gradients. If the assumptions in Equations A17 and A18 are true, then the vertical gradient can be expressed by combining Equation A30 with Equations A17 and A32:

$$
g_{2}=\frac{f(d e f)}{d e f} \phi_{v}
$$

Therefore we can express the angle $\beta$ between the planar surface and the horizontal plane as a function of the deformation by substituting Equations A33 and A17 in Equation A29:

$$
\beta=\arccos \left(\frac{\frac{f(\text { def })}{\operatorname{def}} \phi_{\mathrm{v}}}{\sqrt{f(\text { def })^{2}+1}}\right) .
$$

If we consider that the ratio between $f(d e f)$ and $\operatorname{def}$ is a decreasing function of def, as the results of Experiments 1,2,3, and 4 of the present article indicate, then the numerator of the term in brackets in Equation A34 decreases with the deformation. Since $f($ def $)$ is an increasing function of def (Domini et al., 1995), the whole term in brackets of Equation A34 decreases with the deformation. Therefore, considering that the arc cosine function is a decreasing function, the derived $\beta$ increases as the deformation increases. 


\section{Appendix B}

\section{Reliability Measures}

Reliability measures were calculated for each observer as the standard deviation of the adjustments relative to the mean (Norman, Todd, Perotti, \& Tittle, 1996) and were averaged in each condition of Experiments 1 through 6.

\section{Experiment I}

\begin{tabular}{cllll}
\hline & \multicolumn{4}{c}{ Slant } \\
\cline { 2 - 5 } Depth $(\mathrm{cm})$ & 1 & 2 & 3 & 4 \\
\hline 9.98 & .38 & .43 & .44 & .43 \\
19.96 & .25 & .30 & .35 & .30 \\
\hline
\end{tabular}

\section{Experiment 2}

\begin{tabular}{cccccc}
\hline \multicolumn{7}{c}{ Mean slant } \\
\hline 1 & 1.22 & 1.44 & 1.60 & 1.82 & 2.22 \\
\hline .18 & .30 & .30 & .31 & .29 & .27 \\
\hline
\end{tabular}

Experiment 3

\begin{tabular}{cccc}
\hline & \multicolumn{3}{c}{ Mean slant } \\
\cline { 2 - 4 } Condition & 1.22 & 1.605 & 1.825 \\
\hline Surface & .26 & .27 & .25 \\
Cloud & .22 & .23 & .24 \\
\hline
\end{tabular}

Experiment 4

\begin{tabular}{lccccccc}
\hline & \multicolumn{7}{c}{ Slant difference } \\
\cline { 2 - 8 } Axis & -1.21 & -.77 & -.44 & 0 & .44 & .77 & 1.21 \\
\hline Behind & .29 & .32 & .26 & .37 & .32 & .40 & .26 \\
Front & .34 & .29 & .31 & .37 & .35 & .27 & .32 \\
\hline
\end{tabular}

Experiment 5

\begin{tabular}{lcccc}
\hline & \multicolumn{4}{c}{ Probe pair } \\
\cline { 2 - 5 } Slant & 0,1 & 2,3 & 0,3 & 1,2 \\
\hline 1 & .16 & .11 & .32 & .31 \\
1.44 & .20 & .16 & .29 & .32 \\
2.21 & .20 & .26 & .20 & .18 \\
\hline
\end{tabular}

Experiment 6

\begin{tabular}{ccc}
\hline & \multicolumn{2}{c}{$g_{1,1}$} \\
\cline { 2 - 3 }$g_{2,1}$ & 0.0 & 2.0 \\
\hline 0.0 & .39 & .70 \\
2.0 & .35 & .20 \\
\hline
\end{tabular}

